\title{
PISCO: The PMAS/PPak Integral-field Supernova Hosts Compilation
}

\author{
L. Galbany ${ }^{1}(1)$ J. P. Anderson ${ }^{2}$, S. F. Sánchez ${ }^{3}$, H. Kuncarayakti ${ }^{4,5}$ (), S. Pedraz ${ }^{6}$, S. González-Gaitán ${ }^{7}$, V. Stanishev ${ }^{8}$ (1), \\ I. Domínguez ${ }^{9}$, M. E. Moreno-Raya ${ }^{6}$ (D) , W. M. Wood-Vasey ${ }^{1}$ (D) , A. M. Mourão ${ }^{7}$, K. A. Ponder ${ }^{10}$ (iD) C. Badenes ${ }^{1}$ (iD , M. Mollá ${ }^{11}$, \\ A. R. López-Sánchez ${ }^{12,13}$, F. F. Rosales-Ortega ${ }^{14}$ (DD , J. M. Vílchez ${ }^{15}$, R. García-Benito ${ }^{15}$, and R. A. Marino ${ }^{16}$ (ID \\ ${ }^{1}$ PITT PACC, Department of Physics and Astronomy, University of Pittsburgh, Pittsburgh, PA 15260, USA; 1lgalbany@ pitt.edu \\ ${ }^{2}$ European Southern Observatory, Alonso de Cordova 3107 Casilla 19001-Vitacura-Santiago, Chile \\ ${ }^{3}$ Instituto de Astronomía, Universidad Nacional Autónoma de México, A.P. 70-264, 04510 México, D.F., Mexico \\ ${ }^{4}$ Finnish Centre for Astronomy with ESO (FINCA), University of Turku, Väisäläntie 20, FI-21500 Piikkiö, Finland \\ ${ }^{5}$ Tuorla Observatory, Department of Physics and Astronomy, University of Turku, Väisäläntie 20, FI-21500 Piikkiö, Finland \\ ${ }^{6}$ Centro Astronómico Hispano-Alemán (CSIC-MPG), Observatorio Astronómico de Calar Alto, Sierra de los Filabres-E-04550 Gérgal, Almería, Spain \\ ${ }^{7}$ CENTRA-Centro de Astrofísica e Gravitação, Instituto Superior Técnico, Av. Rovisco Pais 1, 1049-001, Lisbon, Portugal \\ ${ }^{8}$ Department of Physics, Chemistry and Biology, IFM, Linköping University, SE-581 83 Linköping, Sweden \\ ${ }^{9}$ Dpto. Física Teórica y del Cosmos, Universidad de Granada, E-18071 Granada, Spain \\ ${ }^{10}$ Berkeley Center for Cosmological Physics, Campbell Hall 341, University of California, Berkeley, CA 94720, USA \\ ${ }^{11}$ Departamento de Investigación Básica, CIEMAT, Avda. Complutense 40, E-28040, Madrid, Spain \\ ${ }_{12}$ Australian Astronomical Observatory, P.O. Box 915, North Ryde, NSW 1670, Australia \\ ${ }^{13}$ Department of Physics and Astronomy, Macquarie University, NSW 2109, Australia \\ ${ }^{14}$ Instituto Nacional de Astrofísica, Óptica y Electrónica (INAOE), 72840 Tonantzintla, Puebla, Mexico \\ ${ }^{15}$ Instituto de Astrofísica de Andalucía (IAA/CSIC), Glorieta de la Astronomía s/n Aptdo. 3004, E-18080 Granada, Spain \\ ${ }^{16}$ ETH Zürich, Department of Physics, Wolfgang-Pauli-Str. 27, 8093 Zürich, Switzerland \\ Received 2017 December 19; revised 2018 February 1; accepted 2018 February 4; published 2018 March 13
}

\begin{abstract}
We present the PMAS/PPak Integral-field Supernova hosts COmpilation (PISCO), which comprises integral field spectroscopy (IFS) of 232 supernova (SN) host galaxies that hosted $272 \mathrm{SNe}$, observed over several semesters with the $3.5 \mathrm{~m}$ telescope at the Calar Alto Observatory (CAHA). PISCO is the largest collection of SN host galaxies observed with wide-field IFS, totaling 466,347 individual spectra covering a typical spatial resolution of $\sim 380 \mathrm{pc}$. Focused studies regarding specific SN Ia-related topics will be published elsewhere; this paper aims to present the properties of the SN environments, using stellar population (SP) synthesis, and the gas-phase interstellar medium, providing additional results separating stripped-envelope SNe into their subtypes. With 11,270 H II regions detected in all galaxies, we present for the first time a statistical analysis of $\mathrm{H}$ II regions, which puts $\mathrm{H}$ II regions that have hosted SNe in context with all other star-forming clumps within their galaxies. SNe Ic are associated with environments that are more metal-rich and have higher $\mathrm{EW}(\mathrm{H} \alpha)$ and higher star formation rate within their host galaxies than the mean of all H II regions detected within each host. This in contrast to SNe IIb, which occur in environments that are very different compared to other core-collapse SNe types. We find two clear components of young and old SPs at SNe IIn locations. We find that SNe II fast decliners tend to explode at locations where the $\Sigma_{\text {SFR }}$ is more intense. Finally, we outline how a future dedicated IFS survey of galaxies in parallel to an untargeted $\mathrm{SN}$ search would overcome the biases in current environmental studies.
\end{abstract}

Key words: H II regions - supernovae: general - techniques: spectroscopic

Supporting material: machine-readable tables

\section{Introduction}

Supernovae $(\mathrm{SNe})$ are the main drivers of chemical enrichment of the interstellar medium (ISM) in galaxies (Matteucci \& Greggio 1986; Martig \& Bournaud 2010) by propelling the elements created in the interior of their progenitor stars and during explosion (Hoyle \& Fowler 1960; Woosley \& Weaver 1986), and are also one of the main producers of dust grains (Todini \& Ferrara 2001; Bianchi \& Schneider 2007). However, the ultimate link between different kinds of SN explosions and their progenitor systems is far from being identified, and it is also key to our understanding of stellar evolution since various models predict different end paths for stars in a range of masses, metallicities, and ages.

Although SNe Ia are the most precise extragalactic distance indicator to date (Betoule et al. 2014; Scolnic et al. 2017), an exact understanding of the progenitor systems and explosion mechanism of $\mathrm{SNe}$ Ia remains elusive. There is a wide consensus in the community that their progenitors are low-mass stars $\left(1<M<8 M_{\odot}\right.$; Becker \& Iben 1980) that evolve to form degenerate carbon-oxygen $(\mathrm{C} / \mathrm{O})$ white dwarfs (WDs) with masses in the range of $0.5<M<1.1 M_{\odot}$ (Dominguez et al. 1999). A WD in a binary system can accrete mass from its companion star and, under certain conditions, increase its mass to the point where thermonuclear reactions can ignite in its center to completely disrupt the star (Hoyle \& Fowler 1960). Some heterogeneities in the observed properties of SNe Ia can be attributed to differences in the nature of the companion star (another WD or non-degenerate donor star), the actual mass (below or close to the Chandrasekhar limit) of the WD at the explosion (Maoz et al. 2014), or in differences in how the explosion initiates and propagates (as a detonation or a deflagration).

On the other hand, single-star evolution models predict that massive stars $\left(M>8 M_{\odot}\right)$ form a heavy iron core $4-40 \mathrm{Myr}$ after their birth, which gravitationally collapses into a neutron star or a black hole, triggering the explosive ejection of the star's outer envelope and producing a core-collapse supernova 
(CCSN; Bethe et al. 1979; Arnett et al. 1989). Type II SNe are the most common CCSNe. They retain their external $\mathrm{H}$ layer prior to explosion, and have also proved to be useful distance indicators (e.g., de Jaeger et al. 2017). Progenitor detections in pre-explosion images have constrained the initial mass to be between 8.5 and $16.5 M_{\odot}$, the most viable candidates being red supergiants (RSGs; Smartt 2015). Historically, SNe II have been divided into two families, plateau (IIP) and linear (IIL), depending on their post-maximum brightness decay rate, but current evidence have probed this separation to be superfluous since all SNe II cover a continuous range of decay rates (Anderson et al. 2014; Sanders et al. 2015; Galbany et al. 2016b). SNe IIn show narrow lines in their spectra resulting from the interaction between the ejecta and circumstellar matter (CSM). Although only a few progenitor detections have been reported (Gal-Yam \& Leonard 2009), their progenitors could be less massive than normal SNe II (Anderson et al. 2012; Habergham et al. 2014).

A small fraction of CCSNe are stripped of a fraction ( $\mathrm{SNe}$ IIb) or all (SNe Ib) of their $\mathrm{H}$ and even He (SNe Ic) outer layers before explosion. The reason behind this sequence of different levels of mass loss could be the initial zero-age main-sequence mass, metal content, rotation, or interlayer mixing of the progenitor. Two configurations may plausibly explain their nature. In the single-star scenario, the best candidates are massive $\left(>25-30 M_{\odot}\right)$ Wolf-Rayet stars that have been stripped of their envelopes by strong-line driven winds, which are dependent on metallicity (Vink et al. 2001; Crowther 2007). The other possibility is lower-mass stars in binary systems that transfer the outer envelopes through interaction with the companion (Podsiadlowski et al. 1992). In this latter case, the progenitor does not need to be as massive or as young as WR stars. From the six stripped-envelope SN progenitors detected in pre-explosion images, five were $\mathrm{SNe} \mathrm{Ilb}$ (e.g., Maund et al. 2004; Folatelli et al. 2014), all consistent with having binary progenitors, and only one $\mathrm{SN} \mathrm{Ib}$, which was constrained to be a $<25 M_{\odot}$ star that is also in a binary system (Bersten et al. 2014; Kuncarayakti et al. 2015a). Therefore, questions such as the role of binarity, strong-line driven winds, and/or non-steady eruptive mass loss in determining $\mathrm{SN}$ type remain unanswered.

Probing the connection of observed SN properties to a variety of possible progenitor stars has been a topic of interest in astrophysics for decades, but only for a few objects with preexplosion Hubble Space Telescope imaging available have a direct connection been possible. Other methods include the analysis of SN spectra in the nebular phase ( $>200$ days postexplosion) when the elements in the internal layers of the progenitor become optically thin to be detected (Kuncarayakti et al. 2015b; Jerkstrand 2017).

The study of the SN environment, although not a direct approach, has been able to find trends and put constraints by finding different degrees of association for $\mathrm{SN}$ types with a range of host environment properties (see Anderson et al. 2015 b for a review). In this effort, integral field spectroscopy (IFS) has proven useful for both studies of individual nearby galaxies (Krühler et al. 2017) and of statistical samples of SN host galaxies in the nearby universe. Seminal works, such as by Stanishev et al. (2012), showed the power of this approach, and now IFS is the established approach for SN environmental studies (Kuncarayakti et al. 2013a, 2013b, 2017; Rigault et al. 2013; Galbany et al. 2014, 2016a, 2016c, 2017; Lyman et al. 2018).

This paper builds on previous work on SN environments within the Calar Alto Legacy Integral Field Area (CALIFA; Sánchez et al. 2016) survey and presents the PMAS-PPak Integral-field Supernova hosts COmpilation (PISCO) consisting of IFS data of 232 galaxies observed with PMAS/PPak and the $3.5 \mathrm{~m}$ CAHA telescope using the same instrumental configuration. Observations of 100 galaxies are presented here for the first time. PISCO is an effort that responds to the need to increase the number of SN host galaxies observed with PMAS/ PPak both (i) to construct statistical samples for SN subtypes not well-represented in previous works and (ii) to complete the CALIFA DR3 sample with objects in the low-mass regime, decreasing the host galaxy luminosity bias and probing a larger range in host stellar populations (SPs). Studies regarding specific SN Ia-related topics will be published elsewhere; this paper aims to present the CCSN sample and update past results on the correlation between SN type and star formation and the metallicity of its environment. In addition, and taking advantage of the large amount of information contained in a single IFS galaxy observation, we performed an $\mathrm{H}$ II region statistical analysis which allows us to compare SN locations with all other $\mathrm{H}$ II regions within their hosts.

In Section 2, we describe the sample and give details on the specific programs that are part of PISCO. Section 3 contains a full explanation of the analysis performed on the datacubes that is used in subsequent sections. In Section 4, we discuss the masses of the galaxies included in PISCO and how the new observations helped reduce the previous existing bias toward high-mass galaxies present in the CALIFA survey. In Section 5, we update previous results presented in Galbany et al. $(2014,2016 c, 2017)$ including all new observations from PISCO. Section 6 contains the results and discussion of a technique presented in Galbany et al. (2016a) that consists of performing $\mathrm{H}$ II region statistics and studying the properties of the $\mathrm{SN}$ parent $\mathrm{H}$ II region in terms of all other $\mathrm{H}$ II regions in the galaxy. In Section 7, we discuss the implications of our results for different types of SN progenitors. In Section 8, we present, for the first time, a correlation found with PISCO data between SN II post-maximum brightness decline and local star formation rate (SFR) intensity. Finally, in Section 9, we give a summary and list all our conclusions, and in Section 10 we outline future directions of our work.

\section{Sample Description}

PISCO puts together IFS observations of SN host galaxies performed with the Potsdam Multi Aperture Spectograph (PMAS; Roth et al. 2005) in PPak mode (Verheijen et al. 2004; Kelz et al. 2006) mounted to the $3.5 \mathrm{~m}$ telescope of the Centro Astronómico Hispano Alemán (CAHA) located at Calar Alto Observatory in Almería, Spain.

PPak consists of a fiber bundle of 382 fibers, each with a 2 ." 7 diameter, 331 of which (science fibers) are ordered in a single hexagonal bundle with a filling factor of the field of view (FoV) of 55\% (see Figure 1; Verheijen et al. 2004; Kelz et al. 2006). The remaining fibers are used for sky measurements (36), evenly distributed along a circle beyond the science fibers, and for calibration purposes (15). Most of the observations were done with the V500 grating with 500 lines $\mathrm{mm}^{-1}$, which provides a spectral resolution of $\sim 6 \AA$ and covers the whole optical range from 3750 to $7300 \AA$. A significant fraction 
(77/101) of the galaxies in PISCO were also observed with the higher-resolution V1200 grating, which has 1200 lines $\mathrm{mm}^{-1}$ and covers the range from 3400 to $4750 \AA$ with a resolution of $\sim 2.7 \AA$. For those galaxies, we analyzed a combined cube from both the higher spectral resolution in the range covered by the V1200 grating and the V500 resolution up to $7300 \AA$ A. Each galaxy was observed in three $900 \mathrm{~s}$ exposures. The second and third exposures were shifted by an offset of $\Delta$ (R.A., decl. $)=(-5.22,-4.84)$ and $(-5.22,+4.84)$ arcsec with respect to the position of the first exposure to ensure that every point within the FoV is spectroscopically sampled (see Figure 1, top-left panel). The combination of these three pointings provides wavelength- and flux-calibrated 3D datacubes with $100 \%$ covering factor within a hexagonal FoV of $\sim 1.3 \operatorname{arcmin}^{2}$ with $1^{\prime \prime} \times 1^{\prime \prime}$ pixels, which correspond to $\sim 4000$ spectra per object. For galaxies fainter than $r \sim 16$ mag, each individual exposure was increased to $1200 \mathrm{~s}$.

Around half of these observations (132 galaxies that hosted $154 \mathrm{SNe}$ ) were already presented in Galbany et al. (2014, 2016c, 2017), and were performed mostly in the framework of CALIFA (Sánchez et al. 2012a; Walcher et al. 2014), although more specific details can be found in Appendix A. One of the CALIFA-extension projects described in Sánchez et al. (2016) was the "IFS of core collapse supernova environments in low-mass galaxies," and contributed to the third data release with 14 objects (marked in Table 2 with an " $e$ "). Here we present the entire sample of SN host galaxies from that particular program, together with four new programs focused on SNe Ia, making a total of 100 SN host galaxies. The observing program code, title, and time awarded for each of these five campaigns are summarized in Table 1 .

During the first campaign (semester 15B), we obtained observations of 45 (of the 50 proposed) low-mass $\left(<10^{10} M_{\odot}\right)$ galaxies that hosted CCSNe with well-determined classification: SN types II, IIn, IIb, Ib, and Ic. This project aimed to resolve a bias identified in Galbany et al. (2014) due to the absence of low-mass galaxies in the CALIFA sample. The origin of this bias came from the construction of the sample, which was selected from the Sloan Digital Sky Survey (SDSS) DR7 with two simple cuts on redshift and angular size, hence discarding galaxies that did not cover a significant fraction of the instrument FoV (Walcher et al. 2014). With the addition of these new objects, we were able to update the results presented in past works in terms of different SN subtypes and, in addition, increase the completeness of the SN host galaxy sample from the CALIFA survey with low-mass $\left(<10{ }^{10} M_{\odot}\right)$ galaxies. These $\mathrm{SNe}$ were selected from the Open Supernova Catalog (OSC ${ }^{17}$; Guillochon et al. 2017) following these criteria: (a) SN-projected galactocentric distance lower than 40 arcsec, in order to cover the local SN environment with PMAS/PPak, (b) recession velocity of the galaxy lower than $9000 \mathrm{~km} \mathrm{~s}^{-1}(\sim z<0.03)$, (c) $\log _{10}$ D25 (decimal logarithm of the apparent 25 mag $\operatorname{arcmin}^{-2}$ isophotal diameter) lower than 1.12, which corresponds to galactic radius lower than 40 arcsec, (d) decl. $>0^{\circ}$, and (e) SN light curve publicly or privately available.

Studies regarding specific SN Ia-related topics will be published elsewhere; in this paper, we aim to present the CCSN sample, update past results on the correlation between $\mathrm{SN}$ type

\footnotetext{
17 https://sne.space
}

and star formation and metallicity of its environment, and perform a statistical analysis of the $\mathrm{H}$ II region with the entire PISCO sample. Further details on the other programs can be found in Appendix B.

In Table 2, we list the properties of the 232 galaxies and 272 SNe included in PISCO. This consists of 120 type Ia SNe (including two peculiar, two 91bg-like, six 91T-like, and one 02cx-like), 57 stripped-envelope $\mathrm{SNe}$ (including $19 \mathrm{Ib}, 20 \mathrm{Ic}$, $12 \mathrm{IIb}$, one Ic-BL, four typed as Ibc, and one peculiar), and 95 type II SNe (including 19 IIn). All galaxy parameters in Table 2 were taken from the NASA/IPAC Extragalactic Database $\left(\mathrm{NED}^{18}\right)$ or the SIMBAD Astronomical Database ${ }^{19}$ when no information was available. To reduce the effects of SN classification errors from initial discoveries because they might not be completely accurate, we performed a thorough search in discovery and classification reports from the Astronomers Telegram ${ }^{20}$ and the Transient Name Server $\left(\mathrm{TNS}^{21}\right.$ ), the Asiago (Barbon et al. 1989) and OSC SN catalogs, and in published literature through the NASA Astrophysics Data System $\left(\mathrm{ADS}^{22}\right)$. We tried to keep the most precise subtype classification (e.g., Ia 91bg-like, Ic-BL, etc.) when possible, but we left the more ambiguous classification when sources disagree or when we were not confident with the reclassification.

The two upper panels of Figure 2 show a host galaxy $g-r$ color versus absolute $r$-band magnitudes and an absolute $r$-band magnitude $M_{r}$ versus redshift diagrams, all colored by the source the galaxy comes from. Galaxies from the CALIFA sample are included in gray contours for reference. Galaxy magnitudes were retrieved from SDSS DR14 (modelmag; Abolfathi et al. 2017), but for 46 objects, we took their magnitudes from Pan-STARRS1 DR1 (Chambers et al. 2016; preferentially Kron magnitudes, but PSF when those were not available). These panels clearly show how galaxies included in DR3 (labelled CALIFA+Ext. in Figure 2) lie on top of the CALIFA footprint, with some outliers that come from extension programs not following CALIFA sample cuts. Galaxies from PINGS are larger, brighter, and at lower redshifts than the bulk of galaxies included in PISCO, as shown in the figure.

We have plotted together (in blue) all 100 galaxies presented for the first time here, and it can be seen that those corresponding to the low-mass project (blue points with black edges) are objects at similar redshifts to the CALIFA sample but a few magnitudes fainter, which is explained by their smaller sizes (we did not require a minimum size in our sample selection). On the other hand, a few galaxies extend the redshift range up to 0.09 , which correspond to $\mathrm{SN}$ Ia hosts observed in semesters 16B-17B (see Appendix B). In the lower panel of Figure 2, we show the luminosity distance/ redshift and angular physical scale (inner panel) distributions. The average redshift of all 232 galaxies included in PISCO is 0.0192, which corresponds to an angular scale of $\sim 380 \mathrm{pc} / \operatorname{arcsec}$.

\footnotetext{
18 https://ned.ipac.caltech.edu/

19 http://simbad.u-strasbg.fr/simbad

20 http://www.astronomerstelegram.org

21 https://wis-tns.weizmann.ac.il/

22 http://www.adsabs.harvard.edu
} 

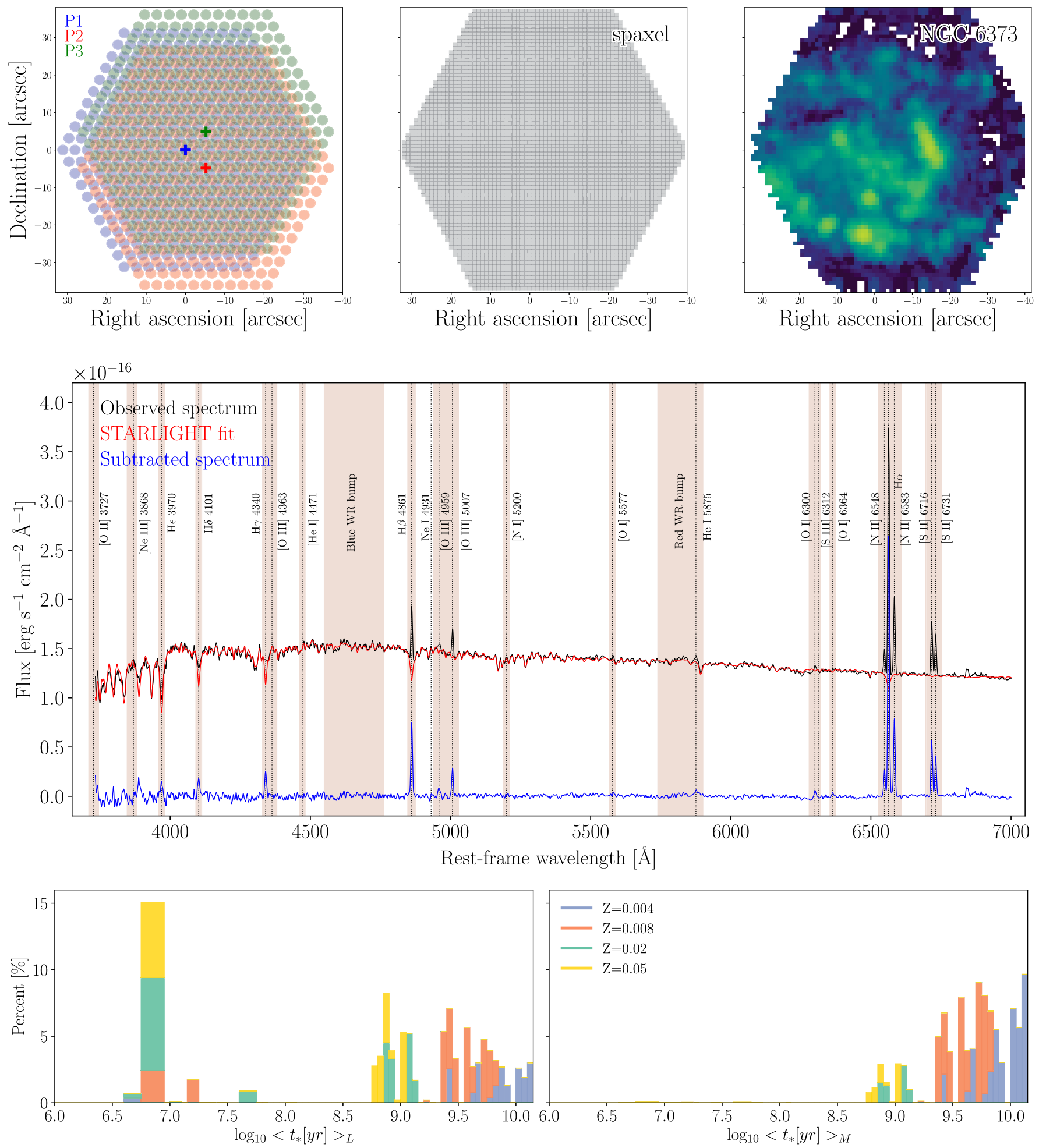

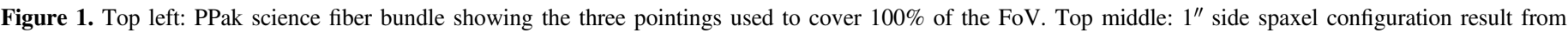

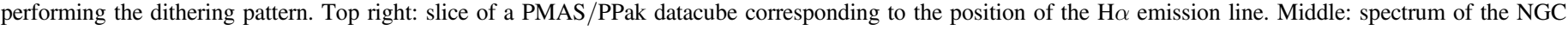

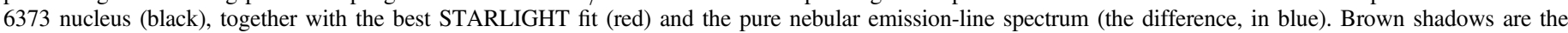

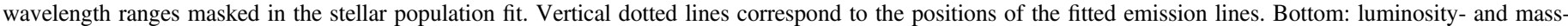
weighted stellar-age distribution of the fit shown in the middle panel. The contributions of the bases with different metallicities are displayed in different colors.

\section{Reduction and Analysis}

\subsection{Reduction}

All data were reduced with the same exact pipeline used for CALIFA DR3 (v2.2; all details can be found in Husemann et al. 2013; García-Benito et al. 2015, and Sánchez et al. 2016). The reduction process is comprised of the following steps:

(i) each set of four FITS files created by the amplifiers of the detector are rearranged into a single frame, which is then bias-subtracted and cleaned of cosmic rays; 
Table 1

Details of the Observing Programs in CAHA Dedicated to Obtaining PMAS/PPak IFS Data of SN Host Galaxies (PI: Galbany)

\begin{tabular}{|c|c|c|c|c|}
\hline Semester & Proposal ID & Time Awarded & Objects Observed & Title \\
\hline $15 \mathrm{~B}$ & H15-3.5-004 & 4 nights & 45 & IFS of core-collapse supernova environments in low-mass galaxies \\
\hline $16 \mathrm{~A}$ & F16-3.5-006 & 5 nights & 21 & Constraining supernova progenitors using the narrow $\mathrm{Na}$ absorption \\
\hline $16 \mathrm{~B}$ & H16-3.5-012 & 2 nights & 9 & Reducing systematic effects in NIR SN Ia standardization \\
\hline 17A & F17-3.5-001 & 3 nights & 12 & Reducing systematic effects in NIR SN Ia standardization II \\
\hline 17B & H17-3.5-001 & 2 nights & 13 & Reducing systematic effects in NIR SN Ia standardization III \\
\hline
\end{tabular}

(ii) relative offsets in the tracing due to flexure are estimated by comparing the continuum and arc-lamp calibration frames, the corresponding wavelength solution is applied to each individual 2D science frame, and all individual spectra are extracted using an optimal extraction algorithm (Horne 1986) and stored in a row-stackedspectrum file;

(iii) flux calibration is performed using a dedicated parallel program, which consists of reobserving two dozen CALIFA early-type galaxies and a set of the standard stars with the PMAS Lens-Array (LArr; B. Husemann et al. 2018, in preparation), and comparing the photometry from these observations to aperture-matched SDSS photometry in the $g$ - and $r$-bands;

(iv) science spectra corresponding to the three dithered exposures were combined into a single frame of 993 spectra. The flux corresponding to the 331 apertures of the fibers for each pointing is measured from skysubtracted SDSS DR7 images in the bands covering the wavelength of our observation, when available. The apertures are shifted over a search box around the nominal coordinates of the pointing, and the best registration is found on the basis of a $\chi^{2}$ comparison, which results in an accurate astrometry with a typical error of $\sim 0$ !" 2 ;

(v) a Galactic extinction correction is applied using dust maps from Schlegel et al. (1998) assuming a Cardelli et al. (1989) extinction law. All cubes in PISCO include this correction in the reduced cubes;

(vi) a flux-conserving inverse-distance weighting scheme is used to reconstruct a spatial image with a sampling of $1^{\prime \prime}$ in a two-step process, first by reconstructing the datacube and estimating the differential atmospheric refraction (DAR) offset, and second by reconstructing the cube again but shifting the position of the fiber at each wavelength against the regular grid according to the DAR offset measured in the first step;

(vii) for the CALIFA DR3 data, when observations with two gratings are available, a combined cube is created, which spans an unvignetted wavelength range of 3700-7140 A. The V1200 data is spatially recentered, flux rescaled, and degraded in spectral resolution to match the V500 data, and the spectra corresponding to each data set in the overlap region is averaged out, weighted by the inverse of the cube error;

(viii) finally, sky-subtracted SDSS DR7 $g$ - and $r$-band images are downsampled to $1^{\prime \prime} /$ pixel (See Figure 1 top-middle panel), and corresponding $g$ - and $r$-band images are also created from the CALIFA datacubes, to match their spectrophotometry as much as possible. Both images are registered using a Discrete Fourier Transform, and the offsets are updated in the CALIFA image and stored in the final 3D datacube. Following this procedure, the absolute spectrophotometric accuracy is better than $3 \%$.

\subsection{Analysis}

The analysis was performed in a similar way to that presented in Stanishev et al. (2012) and Galbany et al. (2014, 2016c, 2017), employing our routines written in IDL $^{23}$ and Python. ${ }^{24}$ The procedures described below were applied to all individual 466,347 spectra included in the 232 datacubes, and also to the 71,654 spectra resulting from a Voronoi-binning tesselation (Cappellari \& Copin 2003) that substituted all spectra with signal-to-noise ratio $(\mathrm{S} / \mathrm{N})$ lower than 20 in a continuum band around $4800 \AA$. In addition, for each galaxy, we extracted a spectrum in a circular aperture $1 \mathrm{kpc}$ in diameter centered on the galaxy core, and an integrated spectrum by summing up all spaxels in the FoV containing galaxy light with $\mathrm{S} / \mathrm{N}$ higher than 1 . In order to exclude light from foreground stars, we constructed 2D masks of point sources from SDSS or Pan-STARRS1 imaging. The 2D spatial configuration of our data allows us to characterize the nature of the emitted light from galaxies. As described below, we mapped regions whose emission is dominated not by ionization from star-forming regions but from the presence of active galactic nuclei (AGNs). We produced two different integrated spectra for those galaxies containing AGNs, the second excluding the above-mentioned region. For SN locations, a circular aperture corresponding to $1 \mathrm{kpc}$ diameter was also extracted. In the case of low $\mathrm{S} / \mathrm{N}$, series of spectra were extracted in apertures centered at the $\mathrm{SN}$ positions and with radii up to $6^{\prime \prime}$, and the spectrum with the smallest aperture with $\mathrm{S} / \mathrm{N}>3$ in the same continuum band was used.

\subsubsection{Stellar Populations}

We used STARLIGHT (Cid Fernandes et al. 2005, 2009) to estimate the fractional contribution of different simple stellar populations (SSPs) with different ages and metallicities to the stellar continuum in the spectra, assuming that the star formation history (SFH) of a galaxy can be approximated as the sum of discrete star formation bursts (see Figure 1, middle and bottom panels). Dust effects, parametrized by $A_{V}^{\text {star }}$, are modeled as a foreground screen with a Fitzpatrick (1999) reddening law assuming $R_{V}=3.1$. From the fits to the integrated spectra, the total stellar mass $\left(M_{*}\right)$ is recovered by combining the mass-to-light ratio of the different SSPs contributing to the best fit. In this work, we selected two different model bases:

\footnotetext{
23 http://www.harrisgeospatial.com/SoftwareTechnology/IDL.aspx

24 https://www.python.org/
} 
Table 2

Properties of the 232 Galaxies and 272 SNe Included in PISCO (Semesters 15B to 17B)

\begin{tabular}{|c|c|c|c|c|c|c|c|c|c|c|}
\hline Galaxy Name & ID & Morphology & Galaxy R.A. & Galaxy Decl. & $z$ & SN & Type & SN R.A. & SN Decl. & Sep. \\
\hline \multicolumn{11}{|c|}{ From CALIFA DR3 } \\
\hline \multirow[t]{3}{*}{ UGC 00005} & 2 & SABbc & 00:03:05.66 & $-01: 54: 49.7$ & 0.024253 & $2000 \mathrm{da}$ & II & 00:03:06.52 & $-01: 54: 41.8$ & 15.1 \\
\hline & & & & & & $20031 q$ & Ia & 00:03:04.02 & $-01: 54: 45.5$ & 24.9 \\
\hline & & & & & & 2016eob & II & 00:03:07.12 & $-01: 54: 42.0$ & 23.2 \\
\hline UGC 00139 & 11 & SAB $(\mathrm{s}) \mathrm{c} ?$ & $00: 14: 31.85$ & $-00: 44: 15.2$ & 0.013219 & $1998 \mathrm{dk}$ & Ia & $00: 14: 32.16$ & $-00: 44: 10.9$ & 6.3 \\
\hline UGC 00148 & 12 & S? & $00: 15: 51.28$ & $+16: 05: 23.2$ & 0.014053 & 2003ld & II & $00: 15: 51.85$ & $+16: 05: 21.6$ & 8.4 \\
\hline NGC 0214 & 28 & $\mathrm{SAB}(\mathrm{r}) \mathrm{c}$ & $00: 41: 28.03$ & $+25: 29: 58.0$ & 0.015134 & $2005 \mathrm{db}$ & IIn & $00: 41: 26.79$ & $+25: 29: 51.6$ & 18.0 \\
\hline NGC 0309 & 34 & $\mathrm{SAB}(\mathrm{r}) \mathrm{c}$ & $00: 56: 42.66$ & $-09: 54: 49.9$ & 0.018886 & $1999 \mathrm{ge}$ & II & $00: 56: 43.76$ & $-09: 54: 43.0$ & 17.7 \\
\hline NGC 0523 & 48 & pec & $01: 25: 20.73$ & $+34: 01: 29.8$ & 0.015871 & $2001 \mathrm{en}$ & Ia & $01: 25: 22.90$ & $+34: 01: 30.5$ & 27.0 \\
\hline NGC 0716 & 65 & SBa? & 01:52:59.68 & $+12: 42: 30.5$ & 0.015204 & 2017 fqo & II & 01:53:01.51 & $+12: 42: 46.0$ & 30.9 \\
\hline
\end{tabular}

(This table is available in its entirety in machine-readable form.)

(i) to fit all individual and Voronoi spectra, we chose a selection of 66 components with 17 different ages (from $1 \mathrm{Myr}$ to $18 \mathrm{Gyr})$ and four metallicities $(0.2,0.4,1.0$, and $2.5 Z_{\odot}$, where $\left.Z_{\odot}=0.02\right)$ coming from a slightly modified version of the models of Bruzual \& Charlot (2003), ${ }^{25}$ replacing STELIB by the MILES spectral library (Sánchez-Blázquez et al. 2006), Padova 1994 evolutionary tracks, Chabrier (2003) initial mass function (IMF) truncated at 0.1 and $100 M_{\odot}$, with calculations of the TP-AGB evolutionary phase for stars of different mass and metallicity by Marigo \& Girardi (2007) and Marigo et al. (2008).

(ii) to fit all integrated, central, $\mathrm{SN}$ location, and $\mathrm{H}$ II region (see Section 3.3) spectra, we used the "Granada-Miles" (GM) base, which is a combination of the MILES SSP spectra provided by Vazdekis et al. (2010; as updated by Falcón-Barroso et al. 2011) for populations older than $t=63 \mathrm{Myr}$ and the González Delgado et al. (2005) models for younger ages. They are based on the Salpeter (1955) IMF and the evolutionary tracks by Girardi et al. (2000), except for the youngest ages ( $<3 \mathrm{Myr}$ ), which are based on Geneva tracks (Schaller et al. 1992; Schaerer et al. 1993a, 1993b; Charbonnel et al. 1993). The GM base is defined as a regular $(t, Z)$ grid of 248 models with 62 ages spanning $t=0.001-14$ Gyr and four metallicites $\left(Z / Z_{\text {sun }}=0.2,0.4,1\right.$, and 1.5$)$.

The main reason for choosing (i) over (ii) to do the initial analysis was the larger amount of spectra to fit $(\sim 500,000$ over a few thousands) and the lower number of bases (66 over 248), which reduced the amount of computing time. In general, results from both bases are broadly similar to those discussed in González Delgado et al. (2015), although SFHs are smoother in a larger base.

\subsubsection{Gas-phase Emission}

We subtracted STARLIGHT fits from the observed spectra to obtain pure gas emission spectra (see Figure 1, middle panel). We then accurately measure the flux of the most prominent emission lines by means of a weighted nonlinear least-squares fit with a single Gaussian plus a linear term. Errors on the flux measurement were determined from the $\mathrm{S} / \mathrm{N}$ of the line flux and the ratio among the fitted amplitude to the

\footnotetext{
${ }^{25}$ See Bruzual (2007) for more information
}

standard deviation of the underlying continuum. The flux of $\mathrm{H} \alpha$ is a good proxy for the current $(<10 \mathrm{Myr})$ SFR and has been extensively used in the literature (Kennicutt 1998a; Catalán-Torrecilla et al. 2015). Moreover, the intensity ratio of the observed $\mathrm{H} \alpha(6563 \AA)$ and $\mathrm{H} \beta(4861 \AA)$ emission lines, i.e., the Balmer decrement, provides an estimate of the optical extinction due to the dust attenuation. In photoionized nebulae, the intrinsic value of the $I(\mathrm{H} \alpha) / I(\mathrm{H} \beta)$ ratio is 2.86, representative of low-density nebular conditions $\sim 10^{3} \mathrm{~cm}^{-3}$ around a heating source with a typical temperature $T \sim 10^{4} \mathrm{~K}$ and large optical depths (Case B recombination; Osterbrock \& Ferland 2006). Using a Fitzpatrick (1999) extinction law, we obtain an estimate of the color excess $E(B-V)$ due to host galaxy dust, which, adopting the Galactic average value of $R_{V}=A_{V}^{\text {gas }} / E(B-V)=3.1$, can be used to infer $A_{V}^{\text {gas }}$. All optical emission lines previously measured are then corrected for the host galaxy dust extinction. In addition, $\mathrm{H} \alpha$ equivalent widths (EWs) are measured by first dividing the observed spectra by the STARLIGHT fit, and repeating the weighted nonlinear least-squares fit in the normalized spectra. Finally, 2D maps of the emission lines and stellar parameters were constructed. Figure 3 shows the extinction-corrected $\mathrm{H} \alpha$ emission 2D maps for 12 of the new galaxies listed in Table 2. The fluxes and parameters measured in the local $(1 \mathrm{kpc}) \mathrm{SN}$ spectra used in this work are reported in Table 3.

\subsection{H II Region Segmentation}

Wide-field IFS allows SN explosion site parameters to be compared not only to those of the overall host, but to all other SPs found within hosts, therefore exploiting the full capabilities of the data. Following Galbany et al. (2016a), we developed a method to characterize the SN parent cluster in comparison to all other $\mathrm{H}$ II regions in the galaxy, which consists of selecting the closest $\mathrm{H}$ II clump to the $\mathrm{SN}$ (under the assumption that the progenitor was born there) and then comparing such properties to the same parameters measured in all other nebular clusters in the galaxy.

Using our extinction-corrected $\mathrm{H} \alpha$ maps, we selected starforming $\mathrm{H}$ II regions across each galaxy with HIIEXPLORER ${ }^{26}$ (Sánchez et al. 2012b), a package that detects clumps of higher intensity in a map by aggregating adjacent pixels until one of the following criteria is reached: (i) a minimum flux threshold (the median $\mathrm{H} \alpha$ emission in the map), (ii) a relative flux with

\footnotetext{
$\overline{26}$ http://www.caha.es/sanchez/HII_explorer/
} 

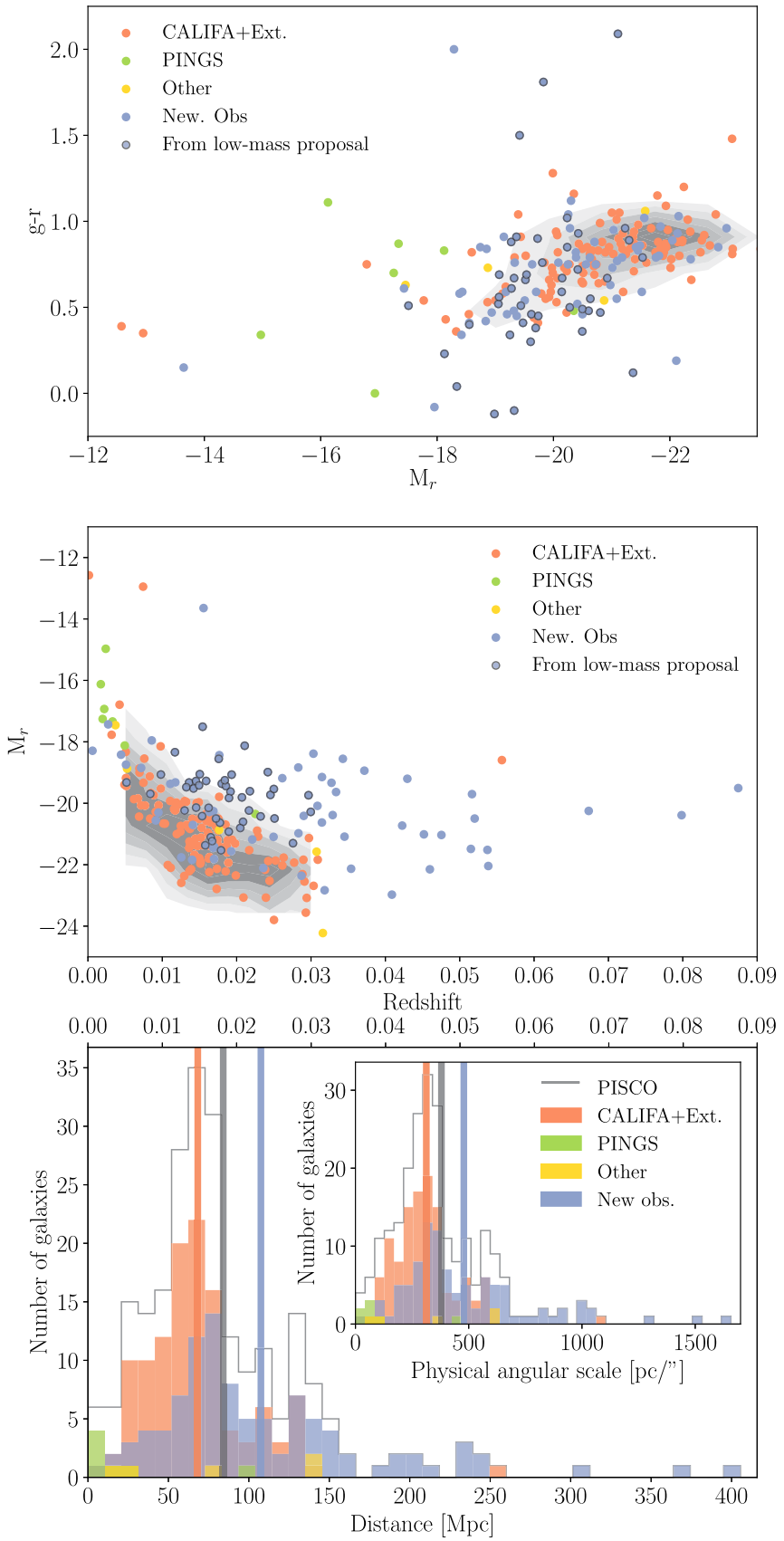

Figure 2. Top panel: $g-r$ color vs. absolute $r$-band magnitude $\left(M_{r}\right)$ diagram. On the background in gray contours are those parameters for the entire CALIFA sample as a reference. Blue dots with the black contour correspond to those objects from PISCO that were observed under the low-mass galaxies program. Middle panel: $M_{r}$ magnitude vs. redshift for all galaxies included in PISCO colored by their source. Note that PINGS galaxies are nearer than CALIFA DR3 galaxies, and galaxies presented here are fainter, and therefore less massive, compared to the CALIFA sample. Bottom panel: distribution of redshift and physical angular scale (pc/spaxel; in the inner panel) for the 232 galaxies in PISCO. Vertical lines are the average redshifts for the CALIFA +Extensions sample (in red), the new observations presented here (in blue), and the entire PISCO sample (in black).

respect to the peak (10\% of the peak flux), or (iii) a radial distance limit $(500 \mathrm{pc})$. The distance limit takes into account the typical size of H II regions of a few hundreds of parsecs (e.g., Gonzalez Delgado \& Perez 1997; Lopez et al. 2011). The code starts with the brightest pixel, and iterates until no peak with a flux exceeding the median $\mathrm{H} \alpha$ emission flux of the galaxy is left. Known caveats of the method are the following: the code does not always select individual $\mathrm{H}$ II regions since the physical scale of a real $\mathrm{H}$ II region could be significantly smaller than the CALIFA pixel size (one to six regions per clump can be expected according to Mast et al. 2014), and the code tends to select regions with similar sizes, although real $\mathrm{H}$ II regions have different sizes.

Once the HII regions were identified, the same analysis described above was performed on the extracted spectra. Spectra with $\operatorname{EW}(\mathrm{H} \alpha)$ lower than $6 \AA$ (justified by a correlation between $\operatorname{EW}(\mathrm{H} \alpha)$ and the fraction of young populations; Sánchez et al. 2013, as shown in the WHAN diagram; Cid Fernandes et al. 2011) or falling in the AGN region in the BPT diagram (Baldwin et al. 1981) according to the Kewley et al. (2001) criterion were discarded to make sure that the emission is caused by ionization from star formation. From all 232 galaxies, the total number of $\mathrm{H}$ II regions that we are left with is 11,270 , which makes an average of $\sim 49$ per galaxy. In Figure 3, we show the resulting $\mathrm{H}$ II regions overplotted on the $\mathrm{H} \alpha 2 \mathrm{D}$ maps for 12 galaxies.

\section{Galaxy Stellar Masses}

Figure 4 shows the distribution of stellar masses, in units of $\log _{10} M_{\odot}$, for various PISCO galaxy subsamples. In solid thick lines, we show the distributions of all SN Ia (in blue) and CCSN (in red) host galaxies in PISCO. All mass measurements are reported in Table 3. As expected, SN Ia hosts have on average larger stellar masses (10.36 dex), as they include the earliest types, compared to CCSN hosts (10.11 dex), which are all late type and also include a selected group of low-mass galaxies.

We also include, as a comparison, shown by the dotted and dashed lines respectively, stellar masses from representative targeted and untargeted SN host galaxy samples from the literature: targeted CCSN hosts of Kelly \& Kirshner (2012); untargeted CCSN hosts from the PTF survey (Stoll et al. 2013) and from Kelly \& Kirshner (2012) compiled from several different surveys; untargeted SN Ia hosts from PTF (Pan et al. 2014); and targeted SN Ia galaxies of Kelly \& Kirshner (2012) and Neill et al. (2009). Finally, we split each PISCO SN Ia and CCSN subsample in two, to study how the representativeness of PISCO has improved compared with the sample presented in G16. We performed two-sample AndersonDarlington (AD) tests to check whether distributions were drawn from the same underlying population. The AD test is a modification of the Kolmogorov-Smirnov (KS) test; whereas the $\mathrm{KS}$ test is more sensitive to the center of distribution, the $\mathrm{AD}$ test is more sensitive to the tails of a distribution,

SN Ia host galaxies presented in G16 have an average stellar mass of $10.58 \mathrm{dex}$ and are representative of galaxies that host $\mathrm{SNe}$ Ia discovered by targeted searches $\left(\mathrm{AD}_{\mathrm{G} 16-\mathrm{Ia}, \mathrm{T}}=0.10\right.$, $\mathrm{AD}_{\mathrm{G} 16-\mathrm{Ia}, \mathrm{U}}=0.02$ ). In this present paper, we add 38 new objects with a lower average stellar mass (10.08 dex). Figure 4 clearly shows that these new objects convert the PISCO SN Ia galaxy sample into one that is compatible with the untargeted SN Ia PTF group $\left(\mathrm{AD}_{\mathrm{PISCO}-\mathrm{Ia}, \mathrm{U}}=0.29\right)$ and far from the targeted distribution $\left(\mathrm{AD}_{\mathrm{PISCO}-\mathrm{Ia}, \mathrm{T}}=1 \mathrm{e}-5\right)$.

For CCSNe, we added 44 objects from the low-mass project with stellar masses significantly lower (9.87 dex), on average, than all of the other CCSN hosts in PISCO (10.32 dex). These two distributions, in orange and dark red in Figure 4, are clearly shifted from each other $\left(\mathrm{AD}_{\text {low,other }}=8 \mathrm{e}-4\right)$. The G16 CCSN 

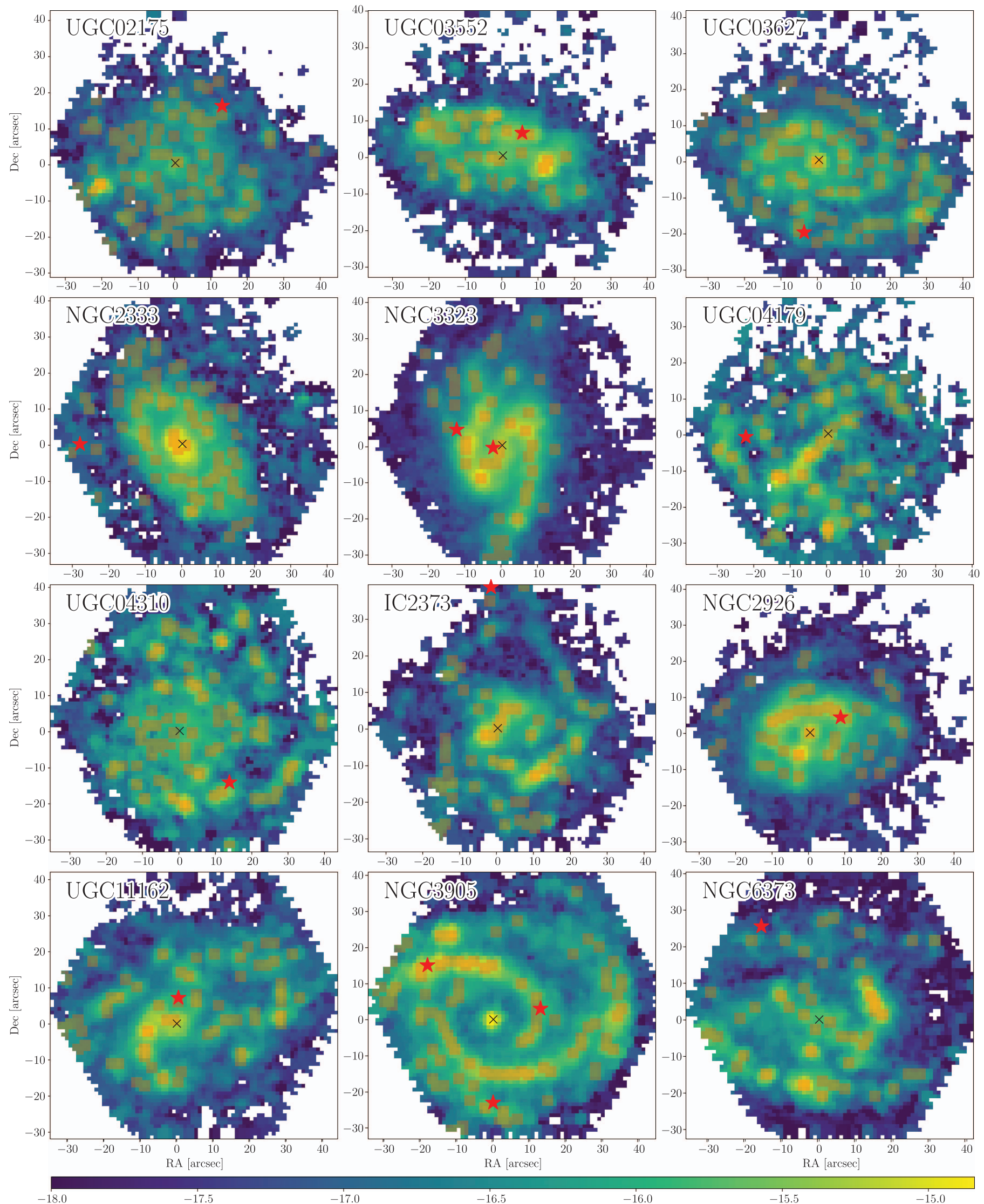

$-17.5$

$-17.0$

$-16.5$

$\log _{10} \mathrm{~F}(\mathrm{H} \alpha)$

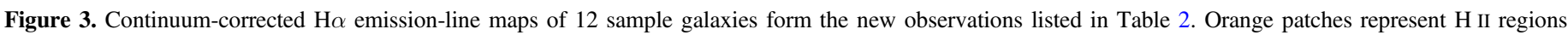

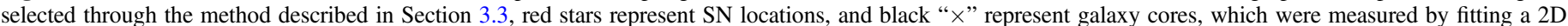
Gaussian function to the collapsed map. 
Table 3

Milky Way and Host Galaxy Extinction-corrected Fluxes and Environment Parameters Measured in the SN Local 1 kpc Diameter Spectra, Together with the Host Galaxy Stellar Mass

\begin{tabular}{|c|c|c|c|c|c|c|c|c|c|c|c|}
\hline SN Name & SN Type & $F(\mathrm{H} \alpha)$ & $\begin{array}{l}F(\mathrm{H} \beta) \\
\quad\left(10^{-1}\right.\end{array}$ & $\begin{array}{c}F(\mathrm{O} \mathrm{III}) \\
\mathrm{erg} \mathrm{s}^{-1} \mathrm{~cm}^{-2}\end{array}$ & ${ }_{-1}^{-1} F(\mathrm{~N} \mathrm{II})$ & $F(\mathrm{~S}$ II $)$ & $\begin{array}{l}\log _{10} \Sigma_{\mathrm{SFR}} \\
\quad(\mathrm{dex})\end{array}$ & $\begin{array}{c}\mathrm{EW}(\mathrm{H} \alpha) \\
(\AA)\end{array}$ & $\begin{array}{c}\left\langle\log _{10}\left(t_{*}[\text { year }]\right)_{\mathrm{L}}\right\rangle \\
(\operatorname{dex})\end{array}$ & $\begin{array}{c}(\mathrm{dex}) \\
\log _{10}(\mathrm{O} / \mathrm{H})_{\mathrm{D} 16}\end{array}$ & $\begin{array}{c}\log _{10}\left(M_{*}\left[M_{\odot}\right]\right) \\
(\operatorname{dex})\end{array}$ \\
\hline $2000 \mathrm{da}$ & II & $28.25(2.00)$ & $9.88(0.68)$ & $1.84(0.14)$ & $9.67(0.70)$ & $8.25(0.36)$ & $-1.5234(0.0319)$ & $32.25(0.50)$ & $8.40(0.14)$ & $8.72(0.02)$ & 11.17 \\
\hline 2003lq & Ia & $6.86(0.50)$ & $2.40(0.19)$ & $1.02(0.10)$ & $2.78(0.20)$ & $3.42(0.18)$ & $-2.1378(0.0326)$ & $24.39(0.90)$ & $8.88(0.06)$ & $8.58(0.02)$ & 11.17 \\
\hline $2016 \mathrm{eob}$ & II & $6.46(0.47)$ & $2.26(0.15)$ & $0.84(0.08)$ & $2.54(0.17)$ & $2.71(0.11)$ & $-2.1642(0.0325)$ & $24.46(0.58)$ & $8.97(0.12)$ & $8.64(0.02)$ & 11.17 \\
\hline $1998 \mathrm{dk}$ & Ia & $80.88(5.88)$ & $28.28(2.09)$ & $14.35(1.04)$ & $24.64(1.82)$ & $29.30(1.52)$ & $-1.6059(0.0327)$ & $34.43(0.32)$ & $8.26(0.12)$ & $8.56(0.02)$ & 10.20 \\
\hline 2003ld & II & $204.00(14.67)$ & $71.34(5.44)$ & $50.32(3.67)$ & $61.00(4.37)$ & $74.04(3.91)$ & $-1.1499(0.0324)$ & $44.31(0.33)$ & $8.21(0.15)$ & $8.55(0.02)$ & 10.43 \\
\hline $2005 \mathrm{db}$ & IIn & $159.60(11.66)$ & $55.79(4.06)$ & $7.17(0.62)$ & $53.50(3.86)$ & $35.14(1.84)$ & $-1.1911(0.0330)$ & $50.81(0.50)$ & $7.90(0.13)$ & $8.83(0.03)$ & 11.16 \\
\hline 1999ge & II & $14.82(1.14)$ & $5.18(0.41)$ & $\ldots$ & $5.67(0.46)$ & $3.21(0.19)$ & $-2.0266(0.0347)$ & $17.24(0.36)$ & $8.92(0.10)$ & $8.91(0.04)$ & 11.01 \\
\hline 2001en & Ia & $3.37(0.24)$ & $1.66(0.10)$ & $1.24(0.10)$ & $1.38(0.10)$ & $2.01(0.09)$ & $-2.8244(0.0327)$ & $5.40(0.42)$ & $9.10(0.14)$ & $8.50(0.01)$ & 10.94 \\
\hline $\begin{array}{c}2017 \text { fqo } \\
\quad \ldots\end{array}$ & II & $14.71(1.23)$ & $5.34(0.36)$ & $6.14(0.51)$ & $5.94(0.35)$ & $\ldots$ & $-2.2223(0.0380)$ & $20.00(1.45)$ & $8.36(0.14)$ & $\ldots$ & 10.77 \\
\hline
\end{tabular}

(This table is available in its entirety in machine-readable form.) 

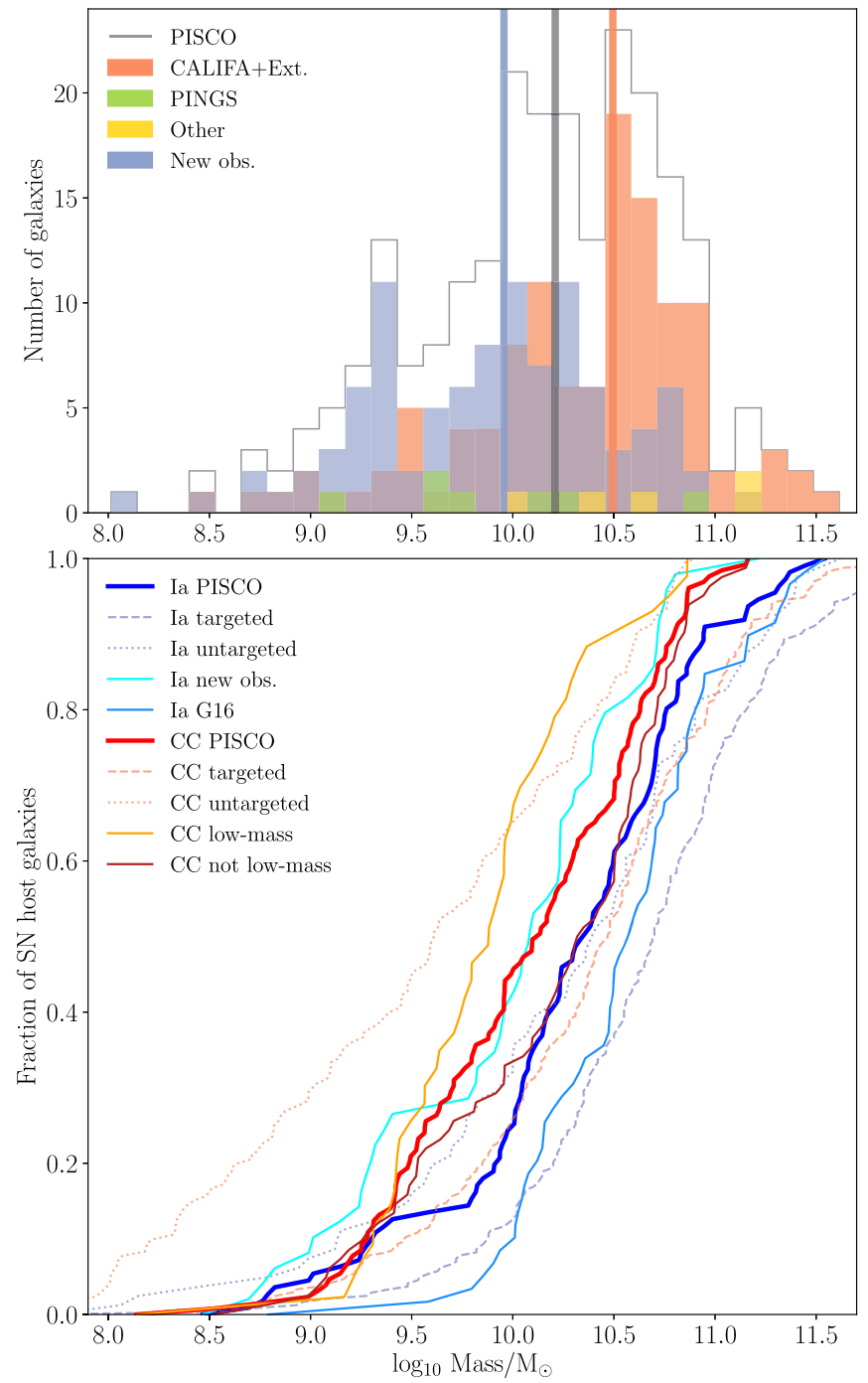

Figure 4. Cumulative distribution of the masses of all PISCO galaxies separated by SN Ia and CCSN hosts. As a comparison, we also show the untargeted (dotted lines) CCSNe hosts from the PTF (Stoll et al. 2013) and from Kelly \& Kirshner (2012) compiled from several different surveys, as well as the targeted (dashed lines) CCSN galaxy samples of Kelly \& Kirshner (2012). We show untargeted SN Ia hosts from PTF (Pan et al. 2014) and targeted SN Ia galaxies of Kelly \& Kirshner (2012) and Neill et al. (2009). This figure clearly shows that the SN targeted surveys are biased toward massive galaxies. While in Galbany et al. (2016c) we showed that the SN host galaxies from CALIFA had masses similar to other targeted searches, SN Ia host galaxies in PISCO follow the untargeted SN Ia host galaxy distribution. However, even with the addition of galaxies observed under the low-mass project (orange distribution), we see that CCSN host galaxy distribution in PISCO is between targeted and untargeted CCSN distributions.

host galaxy sample was already incompatible with the targeted CCSN host compiled from the literature, but was more similar to that than to the untargeted sample $\left(\mathrm{AD}_{\mathrm{G} 16-\mathrm{CC}, \mathrm{T}}=0.01\right.$, $\left.\mathrm{AD}_{\mathrm{G} 16-\mathrm{CC}, \mathrm{U}}=8 \mathrm{e}-6\right)$. The addition of the new observations from the low-mass project shifts PISCO CCSN distribution to lower values, but still it is not compatible with the distribution of CCSN hosts discovered by untargeted surveys $\left(\mathrm{AD}_{\mathrm{PISCO}-\mathrm{CC}, \mathrm{U}}=\right.$ 1e-5), although it is now less compatible with the targeted distribution $\left(\mathrm{AD}_{\mathrm{PISCO}-\mathrm{CC}, \mathrm{T}}=2 \mathrm{e}-5\right)$.

The comparison of PISCO SN host properties with existing data for SN Ia and CCSN hosts leads us to conclude that we have been able to construct an SN Ia host galaxy sample compatible with SN Ia hosts from unbiased untargeted searches. However, we are still far from a representative CCSN galaxy sample, although closer than in previous works. This stresses the importance of continuing to obtain IFS data of galaxies with low masses $-8.0<\log _{10}\left(M\left[M_{\odot}\right]\right)<10.0$ in order to construct a sample of CCSN host galaxies free of biases introduced by targeted SN searches, which tend to discover objects in high-mass metal-rich hosts.

\section{Local Environmental Parameters}

In the following, we study the differences between the stellar and gas-phase parameters measured in the spectra corresponding to the $1 \mathrm{kpc}$ diameter aperture centered at SN locations. We show the normalized cumulative distributions of SFR intensity $\left(\Sigma_{\text {SFR }}\right), \mathrm{H} \alpha$ equivalent width, the average SP age, and the oxygen abundance for the different SN types. In addition, we study how the SFH varies depending on the SN type found at those locations. These results update those presented in Galbany et al. (2014, hereafter G14) and G16, which were obtained with an SN/galaxy sample less than half the size of the sample presented here. The increment in size has also allowed us to further split SNe into subtypes: from the three groups presented in G14 and G16 (Ia, II, and Ibc), we are now able to differentiate between SNe II with signs of interaction (SNe IIn) and other "normal" SNe II (including historical types $\mathrm{L}$ and $\mathrm{P}$ ), and between types $\mathrm{Ib}$, Ic, and IIb independently. However, for consistency with our previous works, we still include the Ibc+IIb group in our plots, which contain SNe that were accurately classified as Ic, Ib, or IIb, together with other stripped-envelope SNe with less accurate classification, such as Ibc or peculiar.

For visualization purposes of the individual measurement errors, when constructing the normalized cumulative distributions shown in Figures 5, 7, and 8, we generated 1000 distributions for each SN subtype by randomly sampling each measurement using a normal distribution centered at the measured value and with the error as $1 \sigma$. In those figures and in Figure 6, we also summarize the results of the two-sample AD tests performed among all distributions. Darker-blue colors represent lower $p$-values, which correspond to distributions having underlying populations that are significantly different from each other, while brighter greenish colors correspond to distributions that are similar to each other. The average values of all parameters for each SN subtype are listed in Table 4.

\subsection{Correlation with Star Formation}

The extinction-corrected $\mathrm{H} \alpha$ flux $F(\mathrm{H} \alpha)$ is broadly used as a proxy for the ongoing SFR (Kennicutt 1998b). CatalánTorrecilla et al. (2015) demonstrated that the $\mathrm{H} \alpha$ luminosity alone can be used as a tracer of the current SFR, even without including UV and IR measurements, if the underlying stellar absorption and dust attenuation effects have been accounted for, which is the case in this work. In order to properly compare results from galaxies at different redshifts, the fairest parameter is the SFR intensity $\left(\Sigma_{\mathrm{SFR}}=\mathrm{SFR} /\right.$ area $)$. Given that the spectra used in this section have the same physical aperture in all cases $(1 \mathrm{kpc})$, this effect has already been taken into account and our SFR measurements are already SFR intensities.

In the left panel in Figure 5, we show distributions of $\Sigma_{\mathrm{SFR}}$ for all SN subtypes. For consistency, we also plotted together the stripped-envelope $\mathrm{SNe}$ distribution $(\mathrm{Ib}+\mathrm{Ic}+\mathrm{IIb}$, in red). The SN Ia distribution shows the lowest average value. Although 

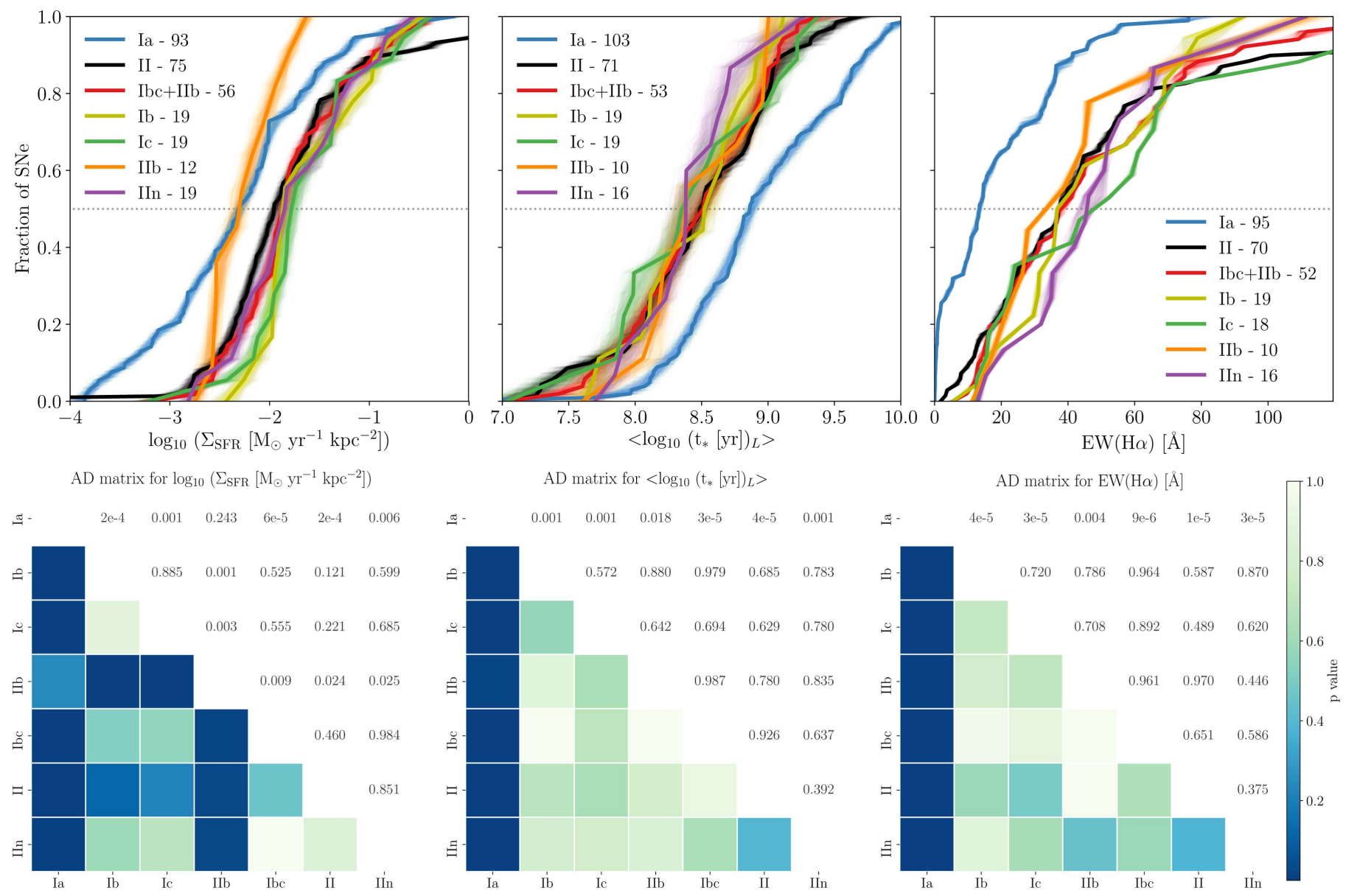

$\mathrm{AD}$ matrix for $<\log _{10}\left(\mathrm{t}_{*}[\mathrm{yr}]\right)_{L}>$

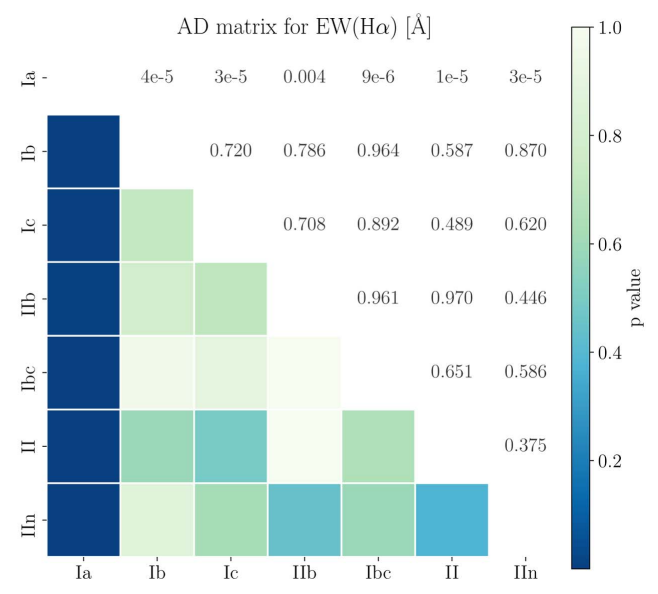

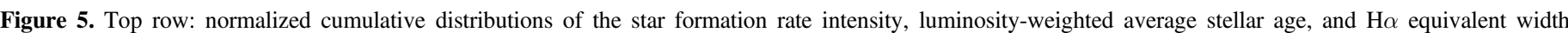

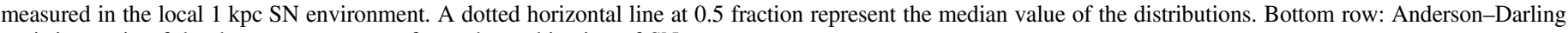
statistic matrix of the three measurements for each combination of SN types.

SNe Ia occur at both high and low $\Sigma_{\mathrm{SFR}}$, it is the only type occurring at locations with $\log _{10} \Sigma_{\mathrm{SFR}}<-3$ dex. Most of the CCSN types are clustered at higher values, but the SN IIb distribution is significantly shifted to lower SFR intensity. Moreover, the steepness of the SN IIb distribution compared to all other distributions also indicates that it is the narrowest, with no environments with $\log _{10} \Sigma_{\mathrm{SFR}} \gtrsim-2$ dex.

\subsection{Correlation with Stellar Age}

The SSP fitting performed with STARLIGHT provides a reconstruction of the SFH. We here calculate the luminosityweighted average stellar age in all $1 \mathrm{kpc}$ local spectra, and show the cumulative distributions in the middle panel of Figure 5 for each SN type. Note that this measurement is built from information of the continuum and does not take into account the gas-phase emission lines at all.

The only significant difference between the distributions, as expected, is that $\mathrm{SNe}$ Ia tend to happen at locations where the average age of the SPs is older. CCSN distributions are clustered at young ages, although the average values are ordered in a sequence from SN Ic, IIn, II, IIb, and Ib in increasing average age.

\subsubsection{Ho Equivalent Width}

While the $\mathrm{H} \alpha$ line luminosity is an indicator of the ongoing SFR traced by ionizing OB stars, the $\mathrm{H} \alpha$ EW measures how strong this is compared to the continuum, which is dominated by old low-mass non-ionizing stars and therefore accounts for most of the galaxy stellar mass. $\mathrm{EW}(\mathrm{H} \alpha)$ can be thought of as an indicator of the strength of the ongoing SFR compared with the past SFR, which reduces with time if no new stars are created, and therefore it is a reliable proxy for the age of the youngest stellar components (Kuncarayakti et al. 2016).

$\mathrm{EW}(\mathrm{H} \alpha)$ can be calibrated to stellar age using SSP models (i.e., Starburst99; Leitherer et al. 1999) assuming single stars born in an instantaneous star formation and distributed in mass according to a particular IMF. Under these assumptions, H II regions are no longer bright in $\mathrm{H} \alpha$ after $\sim 10 \mathrm{Myr}$. However, if either continuous star formation bursts are considered or binary SPs are included, the ionizing populations can survive, further extending the lifetime of $\mathrm{H} \alpha$ emission and therefore the corresponding stellar ages up to $\sim 100 \mathrm{Myr}$ (Eldridge \& Stanway 2009; García-Benito et al. 2011; Zapartas et al. 2017).

$\mathrm{EW}(\mathrm{H} \alpha)$ is the parameter where the clearest differences are found between different CCSN distributions, as can be seen in the right panel of Figure 5. The SN Ic distribution has the highest values, followed by the SNe IIn, II, and Ib distributions. Again, SNe IIb have significantly lower values than other CCSNe. More than $20 \%$ of SNe Ia occur at locations with no or insignificant $\mathrm{EW}(\mathrm{H} \alpha)$, which might mean that there is no gas emission at all, or that the continuum dominates the detected light by a large factor. 

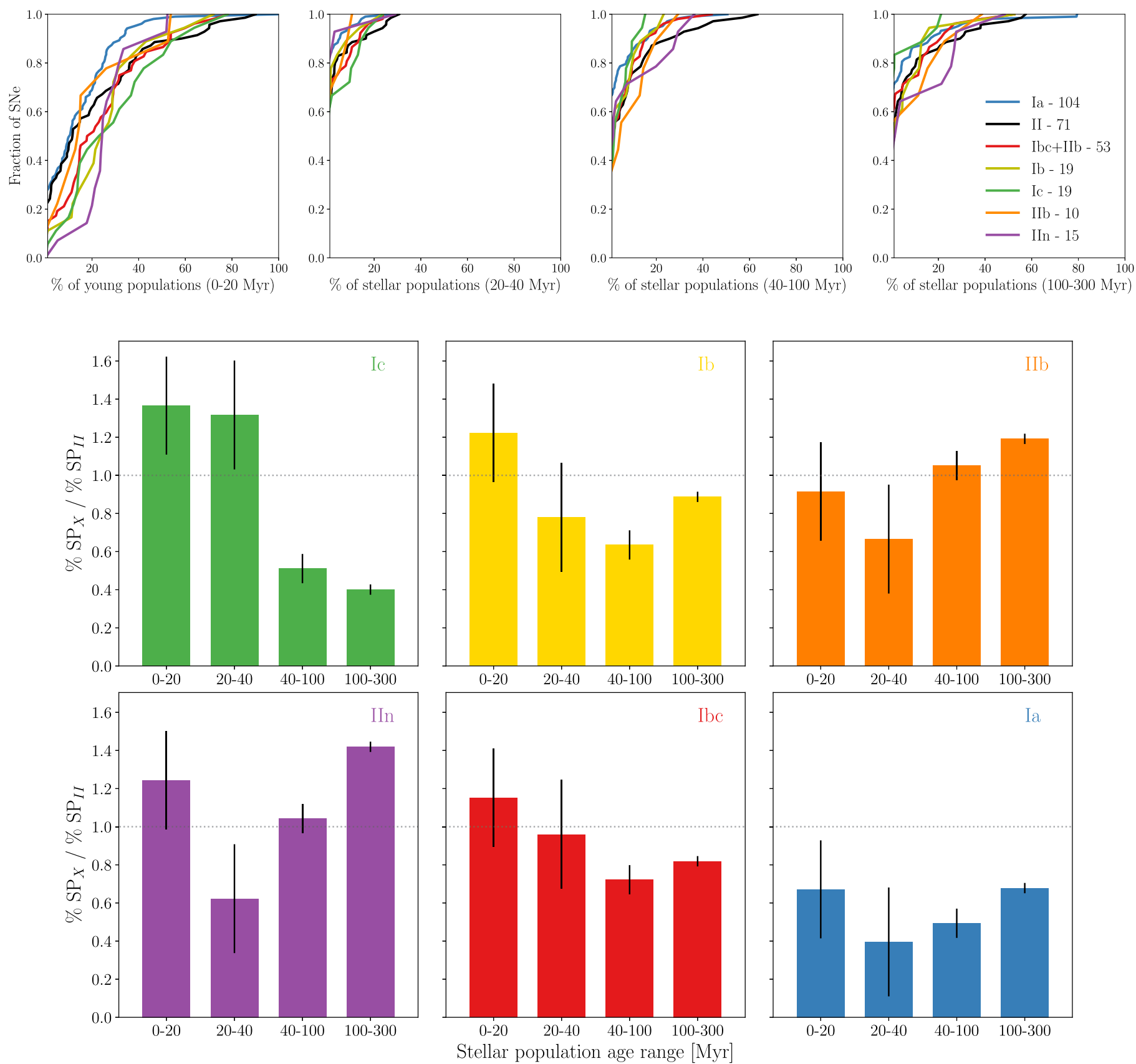

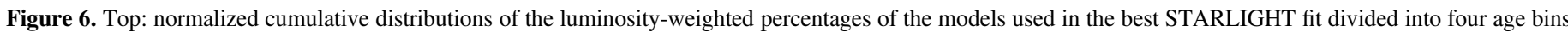

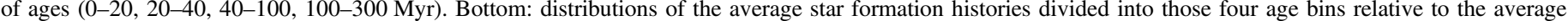
percentages of the SN II distribution (black distribution in the panels above and throughout the paper).

\subsubsection{Star Formation Histories}

Going one step further from the analysis of average stellar ages, and given that SSP fitting allows the reconstruction of the SFH, here we study SPs younger than $300 \mathrm{Myr}$ present at SN locations. From the 248 models available in the "Granada" base used in the SSP fitting, 112 models correspond to populations of interest $(<300 \mathrm{Myr})$. We divided these models into four age bins: 0-20 Myr (40 models), 20-40 Myr (12 models), 40-100 Myr (24 models), and 100-300 Myr (36 models). Note that the Granada base has four different metallicities for each age, so that the number of models with different ages in each age bin is four times smaller.
Figure 6 shows the normalized cumulative distributions of the percentage of models in that particular age bin that were needed to reconstruct the observed spectra in STARLIGHT. The middle panel in Figure 6 shows the AD matrix. The distributions of the youngest age bin (upper-left plot) show a similar sequence to the parameters studied at previous sections: SN Ic locations tend to need more populations in this age bin, followed by $\mathrm{SNe} \mathrm{Ib}$ and $\mathrm{SNe}$ IIn, whose distribution is significantly different from that of SNe II. Again, the SN IIb distribution differs from the other two stripped-envelope $\mathrm{SNe}$, which makes the common $\mathrm{SNe}$ Ibc-IIb distribution (red line) more similar to that of $\mathrm{SNe}$ II than to the individual distributions. Finally, SN] Ia locations require a lower percentage of very young populations, as compared to CCSNe. 
Table 4

Average Values for Each SN Subtype

\begin{tabular}{|c|c|c|c|c|}
\hline SN Type & $\log _{10}\left(\Sigma_{\mathrm{SFR}}\right)$ & $\mathrm{EW}(\mathrm{H} \alpha)(\AA)$ & $\left\langle\log _{10}\left(t_{*}\right)_{L}\right\rangle$ & $12+\log _{10} \mathrm{O} / \mathrm{H}$ \\
\hline $\mathrm{Ia}$ & $-2.317 \pm 0.083$ & $18.296 \pm 1.805$ & $8.935 \pm 0.060$ & $8.643 \pm 0.025$ \\
\hline II & $-1.809 \pm 0.119$ & $56.012 \pm 8.674$ & $8.480 \pm 0.070$ & $8.540 \pm 0.036$ \\
\hline $\mathrm{Ibc}$ & $-1.795 \pm 0.079$ & $47.943 \pm 5.255$ & $8.453 \pm 0.071$ & $8.577 \pm 0.034$ \\
\hline $\mathrm{Ib}$ & $-1.635 \pm 0.121$ & $44.653 \pm 5.439$ & $8.430 \pm 0.103$ & $8.623 \pm 0.036$ \\
\hline Ic & $-1.671 \pm 0.144$ & $58.370 \pm 12.168$ & $8.386 \pm 0.142$ & $8.586 \pm 0.070$ \\
\hline $\mathrm{IIb}$ & $-2.264 \pm 0.096$ & $42.036 \pm 9.178$ & $8.450 \pm 0.140$ & $8.388 \pm 0.072$ \\
\hline IIn & $-1.762 \pm 0.141$ & $48.389 \pm 6.284$ & $8.401 \pm 0.100$ & $8.543 \pm 0.055$ \\
\hline
\end{tabular}

In the other three panels, we note how the SN Ic distribution shifts to the left as the age bin goes to older ages, and SNe IIb and IIn are the subtypes needing additional components of older ages.

The bottom panel of Figure 6 puts together all of the information shown in the upper panels by normalizing the percentage in each bin to the percentage of SNe II (black lines in the upper panels). In this way, given the evidence of RSG being the progenitors of SNe II, we can see how the percentages of other SN types evolve as the age of the SPs gets older. All trends pointed out above are clearly seen in this figure:

1. SNe Ic (green) are above the normalization factor in the first two bins and goes below in the two older bins. This has to be read in terms of "SNe Ic have on average more young SPs of 0-40 Myr than SNe II."

2. On the other hand, the SN Ib (yellow) evolution is flatter in comparison to that of SNe Ic. While in the innermost bin $\mathrm{SNe}$ Ib require more young populations than $\mathrm{SNe}$ II, the average in others bins is lower. In general terms, the behavior of $\mathrm{SNe} \mathrm{Ib}$ is quite similar to that of the $\mathrm{Ibc} / \mathrm{IIb}$ group (in red).

3. $\mathrm{SNe}$ IIb (orange) requires more contribution from populations older than $40 \mathrm{Myr}$ and less from those younger than $40 \mathrm{Myr}$ than SNe II. This is the opposite behavior to $\mathrm{SNe}$ Ic.

4. Another interesting result is that SNe IIn (in purple) have a bimodal behavior, with higher averages both in the innermost and in the older bins and percentages lower than or similar to SNe II in the central two bins.

\subsection{Correlation with Metallicity}

Oxygen is the most abundant metal in the gas phase and exhibits very strong nebular lines at optical wavelengths. This is why it is usually chosen as a metallicity indicator in ISM studies. The most accurate method to measure ISM abundances (the so-called direct method) involves determining the electron temperature of ionized gas, $T_{e}$, which is usually directly measured from the temperature-sensitive intensity ratios of collisionally excited forbidden lines (e.g., [O III] $\lambda 4363 / \lambda 5007$; see Osterbrock \& Ferland 2006), followed by an analysis of the various ionization fractions in the zones of the $\mathrm{H}$ II region that produce optical emission lines. This has been very extensively applied by observers over the past 30 years (e.g., Peimbert \& Costero 1969; Stasińska 1978; Diaz et al. 1987; Vilchez \& Esteban 1996; López-Sánchez \& Esteban 2009). Temperature is anticorrelated with abundance, which makes auroral lines disappear in metal-richer environments, so other strong-line emission methods have to be used instead. While theoretical methods are calibrated by matching the observed line fluxes with those predicted by theoretical photoionization models, empirical methods are calibrated against $\mathrm{H}$ II regions and galaxies whose metallicities have been previously determined with the direct method. Unfortunately, there are large systematic differences between methods, which translate into a considerable uncertainty in the absolute metallicity scale (see López-Sánchez et al. 2012 for a review), while relative metallicities generally agree. The cause of these discrepancies is still not well-understood, although the empirical methods may underestimate the metallicity by a few tenths of dex, while the theoretical methods may overestimate it (Peimbert et al. 2007; Moustakas et al. 2010).

Figure 7 shows the normalized cumulative distributions and AD matrix for the local oxygen abundance measured using the Dopita et al. (2016, D16) calibrator based on photoionization models, but similar relative results stand when the Marino et al. (2013) O3N2 empirical calibrator is used. Both methods have the advantage of being insensitive to flux calibration and extinction due to the small separation in wavelength of the emission lines used for the ratio diagnostics, and neither suffer from DAR (although this is already corrected for when reconstructing the IFS datacube; see Section 3). Although we are not going to strongly rely on the absolute numbers, in the following we will discuss the results in a relative sense.

SNe Ia in PISCO tend to occur in metal-richer environments more so than all other CCSN types; however, the difference is significant only when compared to non-interacting hydrogenrich (type II) $\mathrm{SNe}$ and $\mathrm{SNe}$ IIb. In fact, $\mathrm{SNe}$ IIb occur at significantly metal-poorer locations compared to all other SN types except SNe II and IIn (although $\mathrm{AD}$ is 0.09 and 0.07, respectively). The differences among $\mathrm{SNe}$ Ic, Ib, and II are insignificant. Indeed, as we already noted in Galbany et al. (2016c), the differences among these types arise only when $\mathrm{SNe}$ discovered by untargeted searches are used. Otherwise, the results are biased toward metal-rich galaxies, which are those observed by targeted searches. From the 272 SNe included in PISCO, 205 were discovered in targeted searches and 62 by untargeted surveys. When adding all new observations from PISCO to the same compilation of $\mathrm{SNe}$ from untargeted searches from the literature presented in Galbany et al. $(2016 \mathrm{c}),{ }^{27}$ we recover the same sequence in environmental metallicity from $\mathrm{SNe}$ Ia to broad-line SNe Ic. In the upper panel

\footnotetext{
27 The sample studied here has the following differences with that used in Galbany et al. (2016c): (i) we do not include here those SNe Ia measurements that were performed at the center of their host galaxies but then corrected by an offset of 0.029 dex to account for the difference between local and central values, (ii) we include the measurement of SN 2017ahn from Kuncarayakti et al. (2017), which was a single object from an untargeted search, and (iii) we disregarded measurements of SN environments from other works that are now part of PISCO.
} 

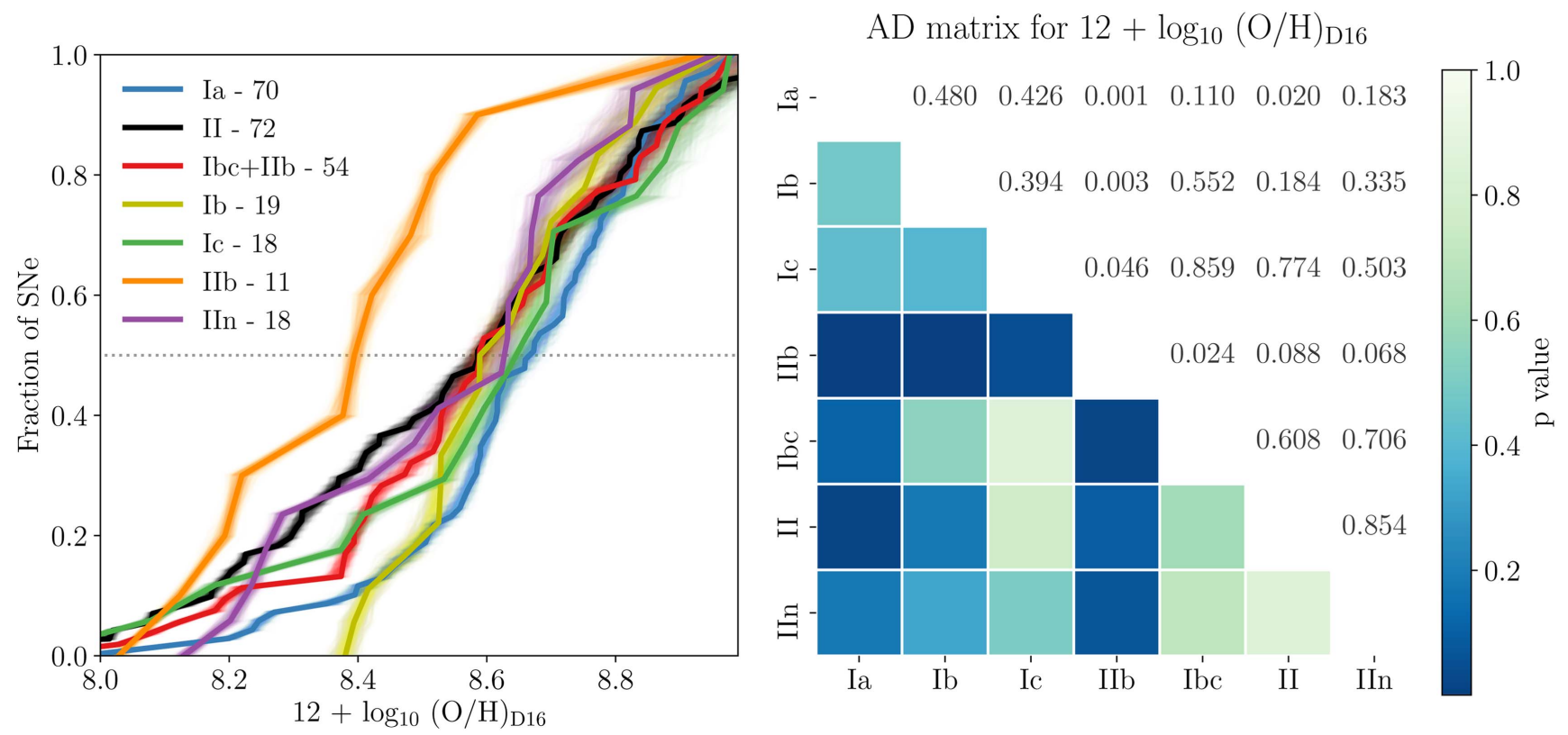

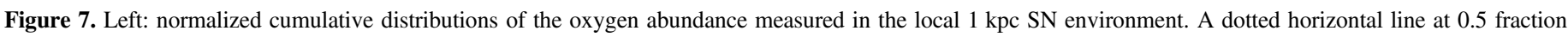
represent the median value of the distributions. Right: Anderson-Darling statistic matrix for each combination of SN types.

of Figure 8, all cumulative distributions are presented. Most of the measurements from the literature were reported in the O3N2 Pettini \& Pagel (2004) scale, so we converted those measured in a different scale to O3N2. Two dotted vertical lines represent the two breaking points used for the division into three bins, and the number ratios of $\mathrm{SNe}$ of different types in those three bins are presented in the lower panel of Figure 8.

Two important facts are shown in these ratios. (i) Even when we have compiled a sample free of objects discovered from targeted searches, it is not volume-limited and other biases can be at play (e.g., our search for CCSNe in low-mass galaxies may be increasing the number of stripped-envelope $\mathrm{SNe}$ at low metallicities, as shown by the Ibc/II ratio in Figure 8, bottom panel), hence differences between $\mathrm{SNe}$ II and strippedenvelope SNe should be approached with caution. This was pointed out recently by Graur et al. (2017) in their recent revision of the LOSS SN sample, which, although it contains targeted $\mathrm{SNe}$, is volume-limited. (ii) Also, despite this enhanced number of stripped-envelope SNe, when all three subtypes are compared a clear sequence from high to low metallicity emerges, Ic-Ib-IIb. Figure 8 shows how the ratio of $\mathrm{Ic} / \mathrm{Ib}$ is highly dependent on metallicity, and also the upper panel shows the SN IIb distribution shifted toward lower metallicities compared to the SN Ib distribution.

\section{H II Region Statistics}

We have seen in Section 5 that differences in the environments of different $\mathrm{SN}$ types can be affected by the sample selection (e.g., the local oxygen abundance shown in Figures 7 and 8). These local measurements are compared among different galaxies but are not normalized out by any global property. For instance, we may estimate a higher local elemental abundance in the environment of an SN exploding in the metal-richest region of a galaxy with low metal abundance than for another SN in the metal-poorest environment in a galaxy that is more chemically evolved.

Pixel statistics techniques, such as the Normalized Cumulative Rank (NCR) method used, e.g., in Anderson et al. (2012) and Lyman et al. (2018) with $\mathrm{H} \alpha$ narrowband imaging as a proxy for SFR, have proved to be useful in finding differences among objects when the samples compared are of large enough size. In Galbany et al. (2016a), we presented a similar approach to the NCR method but using H II regions instead of individual pixels: we constructed galaxy-wide distributions of several parameters measured in all $\mathrm{H}$ II regions of the galaxy and positioned the parent SN H II region values in those distributions. In this way, the locations of the $\mathrm{SN}$ parent regions in these distributions are already normalized and put into the same scale (0-1), allowing useful comparisons among objects occurring in galaxies with a wide range of characteristics. These distributions contain additional information compared to the analysis presented in Section 5. If an SN type was just randomly drawn from the population within galaxies, then one expects a $y$-axis average of 0.5 . An average rank of a particular SN type that significantly deviates from 0.5 suggests that this SN type tends to prefer environments with certain properties within their hosts. We remark that to construct these distributions, IFS data are essential because it allows the extraction of several $\mathrm{H}$ II region integrated spectra from a single datacube; otherwise, a lot of longslit spectra would be needed to get spectra of all $\mathrm{H}$ II regions in a galaxy.

The nearest $\mathrm{H}$ II region from the $\mathrm{SN}$ position was selected by measuring the deprojected distance from the $\mathrm{SN}$ position to the center of all $\mathrm{H}$ II regions, and looking for the lowest value. For the deprojection of distances, we used the $\mathrm{H} \alpha$ velocity map and assumed circular rotation. Given the short lapse (few tens of Myr) between the birth of CCSN progenitor stars and CCSN explosions, these closest regions were considered to be the parent SN H II regions. On the other hand, SNe Ia have long delay times (100 Myr to Gyr), which make it more problematic to associate any given environment directly with the SN progenitor population, as it could have moved a considerable distance before explosion (Sellwood \& Binney 2002). However, the analysis of these explosion sites (see e.g., Rigault et al. 2013; Anderson et al. 2015a; Kelly et al. 2015) can still provide insights into progenitor scenarios by direct comparison 

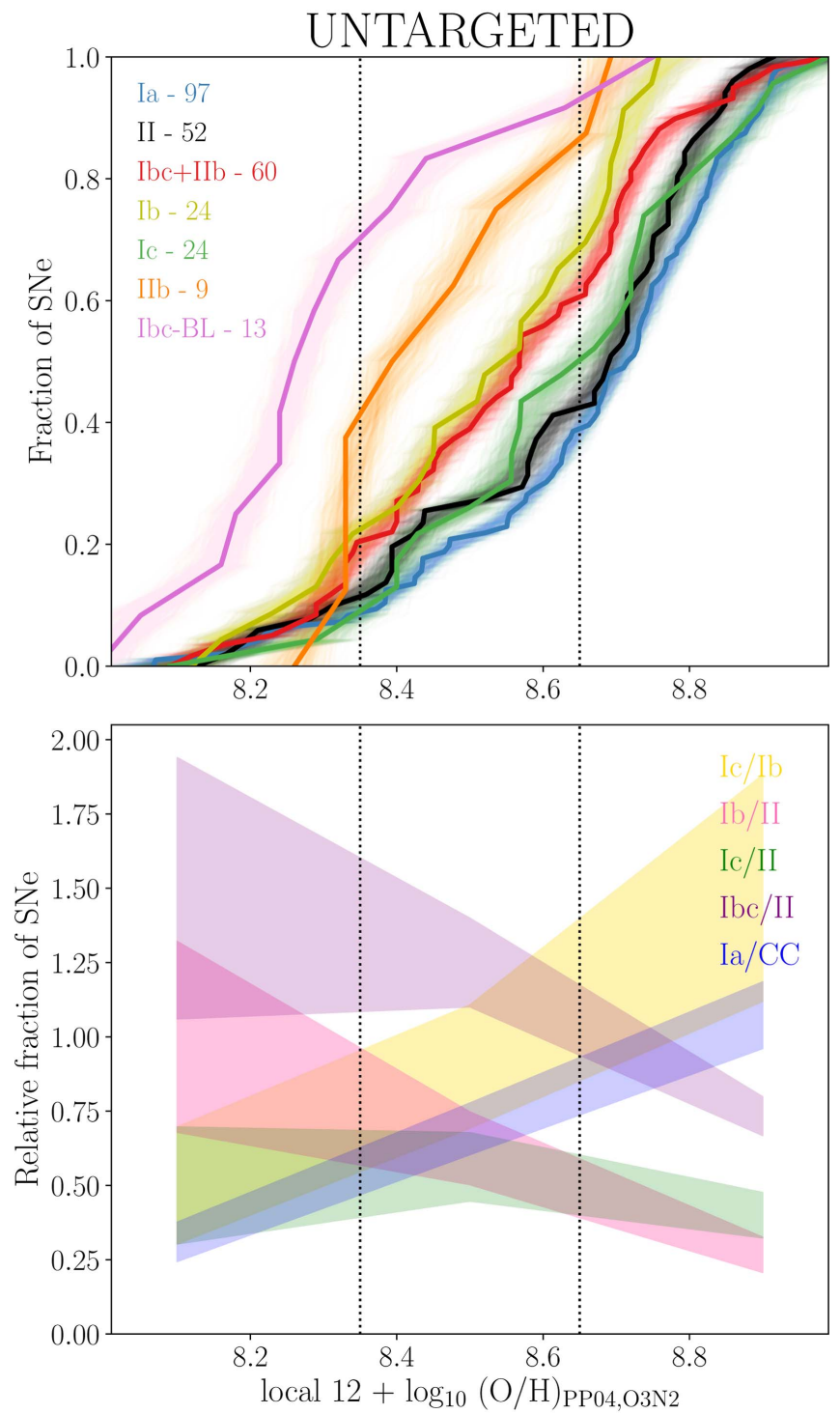

Figure 8. Gas metal abundance measurements at SN locations from PISCO and the literature. Here we only include $\mathrm{SNe}$ that have been discovered by untargeted searches. On top, we show the normalized cumulative distributions for each SN subtype, and at the bottom panel we show how several number ratios evolve as metallicity increase. We calculated the ratio in three bins (limits at 8.35 and 8.65): subsolar, solar, and oversolar. Colored strips connect dispersions measured in each bin.

with observed SN properties. In addition, a big question in SN cosmology is determining to what extent the environment affects systematic uncertainties when determining extragalactic distances. Several correlations have been found using integrated total measurements of galaxy properties, but local environments can provide more consistent correlations (e.g., Rigault et al. 2013 with $\Sigma_{\mathrm{H} \alpha}$; Roman et al. 2017 with $U-V$ color used as a proxy for age).

Figure 9 shows the resulting $\mathrm{H}$ II region distributions of the four parameters studied in Section 5: SFR intensity, $\mathrm{H} \alpha$ equivalent width, light-weighted average stellar age, and oxygen abundance. Each line (in gray) contains measurements in all $\mathrm{H}$ II regions of a single galaxy, and the overplotted (small) symbol represents the value of the SN parent region. Then, for each SN type, we averaged out the measurements at all SN parent $\mathrm{H}$ II regions ( $X$-axis), and the position within their own host galaxy normalized distribution ( $Y$-axis). The resulting averages are shown with larger symbols. Differences in the $X$-axis can be directly compared to the results in Section 5, this time being from measurements performed at the nearest $\mathrm{H}$ II region instead of at the $\mathrm{SN}$ position. However, the differences in the $Y$-axis are giving new information: where the $\mathrm{SN}$ parent cluster is located within all regions of the same galaxy. In this way, an $\mathrm{SN}$ type with higher average values in the $Y$-axis would mean that they systematically occur at higher values, independently of how this parameter compares between the host and other galaxies. This information can only be extracted efficiently from wide-field IFU data. It highlights the usefulness of wide-field IFUs to put the SN parent cluster in context with all other $\mathrm{H}$ II regions in the same galaxy.

We have already shown that while new observations of lowmass galaxies have provided a sample of galaxies more similar to those from unbiased searches (see Section 4), most of the $\mathrm{SNe}$ in PISCO were discovered by biased surveys, and therefore our CCSN sample might not be representative enough of the whole population. This is, for instance, confirmed by our H II region analysis of the SFR.

The upper-left panel of Figure 9 shows that, consistent with our results from Section 5.2.2, parent $\mathrm{H}$ II regions of SNe Ic, Ib, and IIn have slightly higher SFR on average than SNe Ia and IIb. However, it is now clearer that SNe Ic systematically occur at regions with the highest SFR in their host galaxies, compared to both hydrogen-free CCSNe (Ib/IIb) and to SNe II. On the other hand, SNe Ib occur at $\mathrm{H}$ II regions with higher SFR than $\mathrm{SNe}$ II, but these regions are at the same rank within their host galaxies. Again, we find that the most important difference in SFR is between SNe IIb and all other subtypes, the former happening at regions with low SFR and not particularly active within their hosts.

Regarding $\mathrm{EW}(\mathrm{H} \alpha)$, the most significant difference between local and $\mathrm{H}$ II region distributions is the lower $\mathrm{EW}(\mathrm{H} \alpha)$ of $\mathrm{SN}$ IIn parent $\mathrm{H}$ II regions compared to their locations. All SN type averages are within the $45 \%$ and $60 \%$ rank within their hosts, but SN Ic parent regions have both larger $\operatorname{EW}(\mathrm{H} \alpha)$ and are slightly above the other SN types in terms of ranking within their own host galaxies.

All CCSN type parent region averages, except for $\mathrm{SNe} \mathrm{IIb}$, lie on similar stellar ages and within regions that are younger on average than all regions in their hosts. SNe Ia occur on average in regions with older ages, and $\mathrm{SNe}$ IIb fall in between separated from other CCSNe averages.

For oxygen abundances, we find $\mathrm{SN}$ Ia and $\mathrm{SN}$ Ib parent regions have on average higher metallicities than other subtypes, but SNe Ic is the type that tends to occur in metalricher regions within their host galaxies. Again, $\mathrm{SNe} \mathrm{IIb}$ are significantly separated from all other types at lower-metallicity regions both in absolute terms and within their hosts.

In summary, we find $\mathrm{SNe} \mathrm{Ib}$ occur at locations with higher absolute SFR and higher absolute $\mathrm{O} / \mathrm{H}$ compared to Ic locations, but SNe Ic are in higher SFR and $\mathrm{O} / \mathrm{H}$ locations within their host galaxies compared to $\mathrm{SNe} \mathrm{Ib}$, thus strengthening the conclusion that $\mathrm{SNe}$ Ic arise from the highest progenitor metallicity and mass of any $\mathrm{CC}$ type analyzed. We stress here that while any difference in the local values studied in Section 5 may simply just be due to biases in the definition of the sample, when the SN local environment is compared (normalized) to all other environments of the same host galaxy, comparisons among SN types are more fair. This 

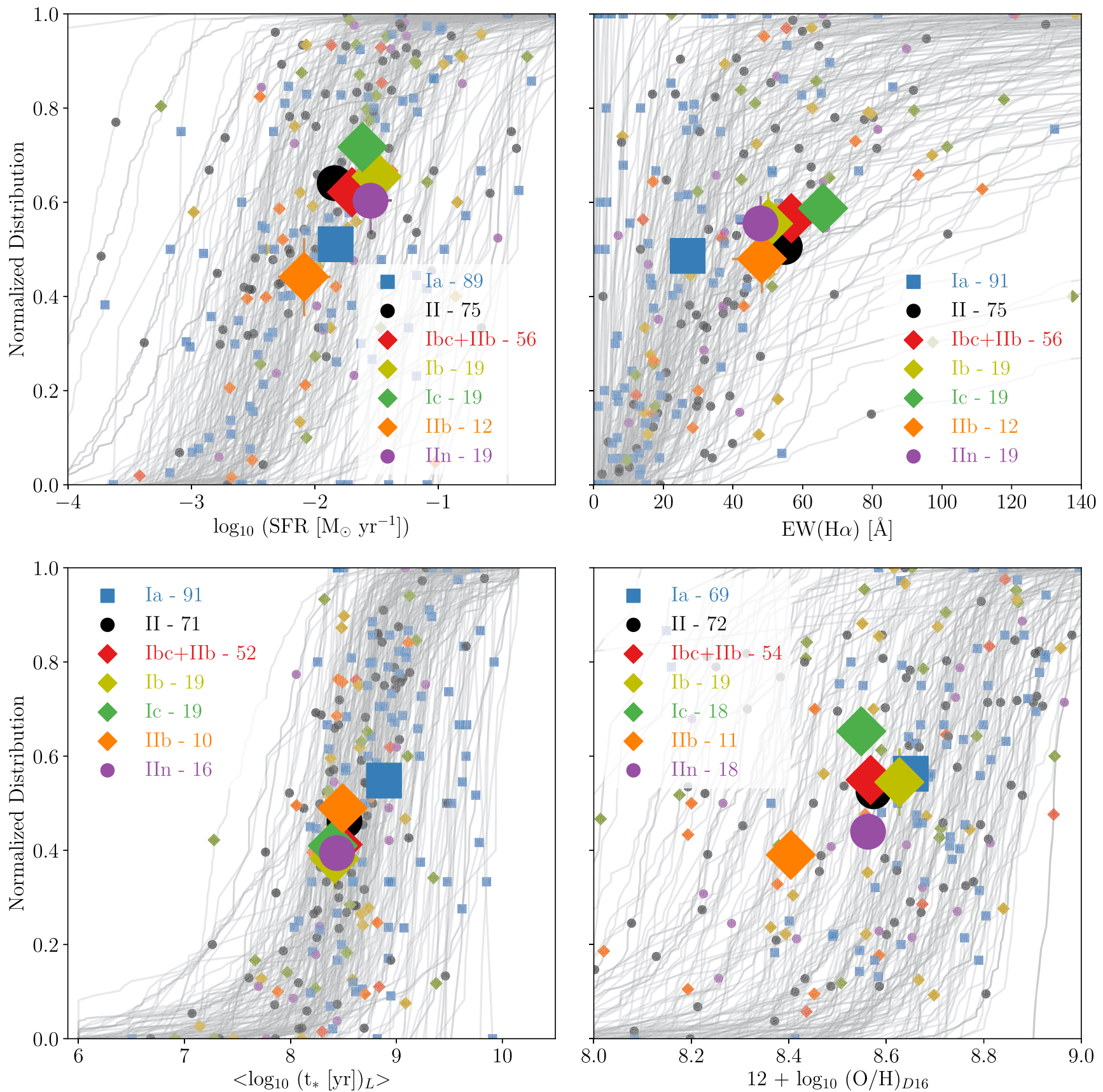

Figure 9. H II region cumulative distributions of SFR, $\mathrm{H} \alpha$ equivalent width, averaged light-weighted stellar age, and oxygen abundance in all galaxies. Each gray line represents one galaxy, while small symbols determine the position and the galaxy parameter at the SN parent H II region. Seven big symbols, in the foreground, represent the average positions of each SN type.

is another demonstration of the power of IFS in studying SN environments.

\section{Implications for Core-collapse Supernova Progenitors}

Single-star evolution models predict that the lower zero-age main-sequence mass limit for a star to produce a CCSN is around $8 M_{\odot}$, which corresponds to a lifetime of $\sim 40 \mathrm{Myr}$ (e.g., Girardi et al. 2000; Heger et al. 2003; Georgy et al. 2009). Given that the majority of massive stars form in binary systems (Sana et al. 2012), more recent theoretical models have considered binaries as an alternative channel for CCSN progenitors. Recently, Zapartas et al. (2017) modeled the effects of binary interactions in the delay time distribution
(DTD) of CCSNe, and demonstrated that the minimum mass for a massive star to explode as an SN can be as low as $4 M_{\odot}$. This lower limit corresponds to longer stellar lifetimes, and the sharp age cutoff for CCSN progenitors at 40 Myr becomes a slow decrease of the DTD, with a tail reaching ages up to $\sim 200$ Myr.

Single RSGs have been found at the locations of SNe II in pre-explosion images, and have been undoubtedly associated as SNe II progenitors (Smartt 2015). For stripped-envelope SNe, the picture is much more confusing. Their progenitors lose the outer layers by some mechanism before explosion and, under the framework of single-star progenitors, Wolf-Rayet (WR) stars have been historically attributed as their most probable 

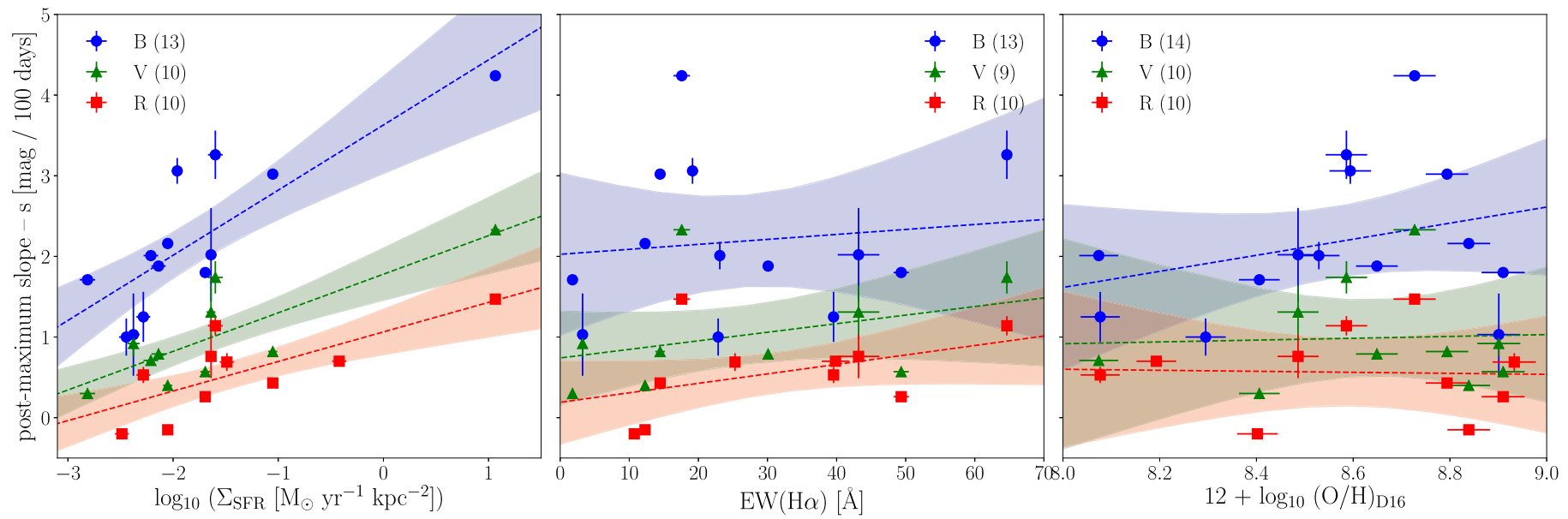

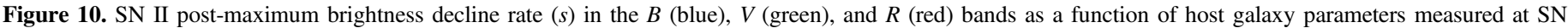

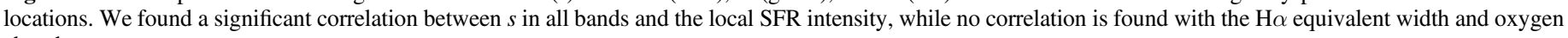
abundance.

progenitors. However, as Smith et al. (2011) pointed out, classical WR stars could not be the only progenitor channel of SNe Ibc, simply because there are not enough WR stars to reproduce their rates and their fraction within all CCSNe ( 30\%; Graur et al. 2017). This is supported by the fact that all six progenitor detections of $\mathrm{SNe} \mathrm{Ibc}$ (one $\mathrm{Ib}$ and five $\mathrm{IIb}$ ) were indeed compatible with binary systems (iPTF13bvn, Eldridge \& Maund 2016; Folatelli et al. 2016; 1993J, Maund et al. 2004; 2008ax, Folatelli et al. 2015; 2011dh, Folatelli et al. 2014; 2013df, Van Dyk et al. 2014; 2016gkg, Tartaglia et al. 2017).

Although these findings would rule against single stars being the exclusive channel, other evidence would favor single-star progenitors. For instance, in binary models, typically a thin layer of hydrogen is left after Roche-lobe overflow, given that it is difficult to remove the helium layer only by mass transfer (Yoon 2017). The total amount of helium is also systematically smaller in single-star models than in binary-star models. Therefore, there may still need to be another mechanism (e.g., metallicity-driven winds) to explain how the remaining He layer in SN Ic progenitors is expelled, or this may simply imply that single-star progenitors are more feasible for SNe Ic (WR stars), while both He-rich subclasses ( $\mathrm{Ib}$, and IIb) are more dominated by lower-mass progenitors in binary systems.

This picture is consistent with our findings. The number ratio $\mathrm{Ic} / \mathrm{Ib}$ (but also Ic/IIb) increases with metallicity (see Figure 5), and their SPs require younger components, as shown in Figures 8 and 6 . In this case, higher metallicity would enable the extra mechanism needed to get rid of the helium layer, and younger populations would indicate younger and higher-mass progenitors. $\mathrm{SNe} \mathrm{Ib}$, on the other hand, have levels of ongoing star formation at their locations similar to SN Ic locations (see Figure 5), but with significantly fewer young SP components and definitely lower metallicities. In the same picture, $\mathrm{SNe} \mathrm{IIb}$ occur at lower SFR environments, with a larger amount of older SSP components (and fewer young SSPs), and at very low metallicity. SNe IIb would perfectly fit in the binary system progenitor scenario with two lower-mass stars, while SN Ib progenitor systems could be either a lower-metallicity single-star case of WR stars (Vink \& de Koter 2005; Crowther 2007; López-Sánchez \& Esteban 2010), a binary system of two stars in different stages of evolution and at higher metallicity (Claeys et al. 2011; Zapartas et al. 2017), or a mixture of both scenarios.
Metallicity is clearly playing a role between $\mathrm{SN}$ Ic/Ib and $\mathrm{SNe} \mathrm{Ib} / \mathrm{IIb}$, but other factors (e.g., progenitor mass or age) might be more important in determining the final fate of hydrogen-rich (II) versus stripped-envelope SNe. The correlation with increasingly younger populations in the sequence Ic $\Rightarrow \mathrm{Ib} \Rightarrow$ II is usually interpreted within the single-star scenario as a difference in progenitor mass and age, but could instead be explained by current binary model predictions (Yoon 2015).

Another interesting result from our analysis is the bimodal SFH found at SN IIn locations. SNe IIn are hydrogen-rich CCSNe that suffer some kind of interaction mostly due to CSM in their close environments as indicated by the narrow Balmer emission seen in their spectra. This suggests that their progenitors have passed episodes of mass-loss activity prior to explosion, by interacting either with their own stripped material by strong winds, or with a companion star in binary systems. As suggested by Taddia et al. (2015), if the mass loss is due to stellar winds, we expect to see a continuity in the observed properties of SNe IIn; however, SNe IIn are the SN type with the most heterogeneous observational properties (even though they only account for $5 \%$ of all CCSNe; Graur et al. 2017), which may translate into a variety of progenitors.

There have been at least four progenitor detections of $\mathrm{SNe}$ IIn in pre-explosion images (1978K, Ryder et al. 1993; 2005gl, Gal-Yam et al. 2009; 2009ip, Mauerhan et al. 2013; Pastorello et al. 2013; 2015bh, Elias-Rosa et al. 2016), and in all cases the object found at the SN IIn location was compatible with very massive and young stars, making the transitional luminous blue variables (LBVs) the most favored progenitors. Although LBVs are usually associated with a transitional phase between a bright $O$ star and a WR, Smith \& Tombleson (2015) examined their locations in the Galaxy and Magellanic Clouds, and proposed that they may be produced in binaries instead.

Taddia et al. (2013) showed that SN IIn environments exhibit a metallicity distribution that closely matches that of SNe II, consistent with what Anderson et al. (2012) found studying the association of SN types with the distribution of the $\mathrm{H} \alpha$ emission (proxy for ongoing SFR) in their host galaxies: the SN IIn distribution was quite similar to that of SN II, indicating that they may come from progenitors with similar stellar masses. Kangas et al. (2017) studied the distribution of massive stars and SNe in the LMC and M33 and also found no correlation between LBV 
and SN IIn NCR distributions, which favored lower-mass progenitors for SNe IIn. Moreover, Kuncarayakti et al. (2017), studying the parent clusters with high-resolution narrow-field IFS, recently found that SN IIn is the type less related to ongoing SFR. RSG progenitors with superwinds have also been proposed to be progenitors of a few individual SNe IIn (e.g., Fransson et al. 2002; Smith et al. 2009). An explanation was given in models by Mackey et al. (2014), where the RSG produces a static shell of CSM confined close to the star, which would produce the narrow lines seen in their spectra.

Our results would explain why detections favored LBVs while SN IIn environments were similar to SN II RSG progenitors: they are very likely to consist of a mixture of very massive and low-mass progenitors. Distributions of SN IIn local environmental parameters (in Figure 5) showed, on average, higher SFR intensity, younger ages, and higher $\mathrm{EW}(\mathrm{H} \alpha)$ than SNe II. This is confirmed when studying their environments within their host galaxies (shown in Figure 9) where similar results are found with respect to SNe II. But the most significant result is that in our SFH analysis (Figure 6), $\mathrm{SNe}$ IIn had a bimodal distribution of stellar ages, and their signal in the innermost and in the oldest SP age bin is higher than that in both the average stripped-envelope SNe and SNe II. Therefore, this would suggest that SNe In come from a mixture of progenitors, a fraction compatible with young and massive progenitors (e.g., LBVs) and probably a higher fraction coming from older progenitor populations (e.g., RSGs), both with the presence of a hydrogen shell close to the star. Unfortunately, there is no IFS data available for a sample of SNe IIn discovered from unbiased surveys, and we were not able to add such distribution of environmental metallicities in Figure 8. This will be the topic of a future work, together with a study of the connection SNe IIn and other interacting SN subtypes (Ibn, Pastorello et al. 2008; Ic/IIn, Kuncarayakti et al. 2018; Ia/IIn, Hamuy et al. 2003).

\section{SN II Light Curve and Environmental Properties Linked}

Hydrogen-rich SNe (type II) have been historically divided into "plateau" (IIP) or "linear" (IIL), depending on the postmaximum brightness decline: type IIP showed a relatively constant brightness for 60-100 days with a sudden decrease plus linear radioactive decay, while type IIL showed a clear decline starting right after maximum light (Barbon et al. 1979). It has been previously claimed (e.g., Arcavi et al. 2010; Faran et al. 2014) that hydrogen-rich SNe II formed two distinct classes (IIP/IIL) powered by different mechanisms (e.g., magnetars, Kasen \& Bildsten 2010; electron capture, Swartz et al. 1991). However, using larger and more complete samples, other works have shown that the post-maximum brightness decline covers a continuum of values from totally flat behavior to quite steep in the $V$ band (Anderson et al. 2014) but also in all other optical bands (González-Gaitán et al. 2015; Sanders et al. 2015; Galbany et al. 2016b; Valenti et al. 2016). Moreover, Anderson et al. (2014) and Valenti et al. (2015) showed that some SNe II, which would have been classified as a typical SN IIL, had this subtle decrease in brightness at the expected epoch (60-100 days) of other SNe IIP. Therefore, hydrogen-rich $\mathrm{SNe}$ are starting to be classified as either fast or slow decliners using different parameterizations (e.g., decline slope after transition from cooling to recombination phases, $s_{2}$, or after peak brightness, $s$, until the end of the recombination phase) and break points. Given that this family spans a
Table 5

Post-maximum Brightness Declines in BVR Bands for the $17 \mathrm{SNe}$ Used in Section 8 and Figure 10

\begin{tabular}{lccc}
\hline \hline SN & $\begin{array}{c}s_{B} \\
{[\mathrm{mag} / 100 \text { days }]}\end{array}$ & $\begin{array}{c}s_{V} \\
{[\mathrm{mag} / 100 \text { days }]}\end{array}$ & $\begin{array}{c}s_{R} \\
{[\mathrm{mag} / 100 \text { days }]}\end{array}$ \\
\hline PTF09cjq & $\ldots$ & $\ldots$ & $0.69(0.11)$ \\
SN 1948B & $2.01(0.17)$ & $\ldots$ & $\ldots$ \\
SN 1994N & $1.00(0.23)$ & $\ldots$ & $\ldots$ \\
SN 1999bg & $2.01(0.04)$ & $0.71(0.03)$ & $\ldots$ \\
SN 1999em & $2.16(0.01)$ & $0.40(0.01)$ & $-0.15(0.01)$ \\
SN 1999gi & $1.80(0.03)$ & $0.57(0.03)$ & $0.26(0.04)$ \\
SN 2003gd & $2.02(0.58)$ & $1.31(0.33)$ & $0.76(0.27)$ \\
SN 2003hg & $3.02(0.04)$ & $0.82(0.02)$ & $0.43(0.02)$ \\
SN 2005au & $3.26(0.30)$ & $1.74(0.20)$ & $1.14(0.12)$ \\
SN 2005dp & $1.25(0.31)$ & $\ldots$ & $0.53(0.10)$ \\
SN 2006be & $1.88(0.04)$ & $0.79(0.01)$ & $\ldots$ \\
SN 2006ee & $1.71(0.04)$ & $0.30(0.03)$ & $\ldots$ \\
SN 2007Q & $1.03(0.51)$ & $0.92(0.16)$ & $\ldots$ \\
SN 2008ij & $3.06(0.16)$ & $\ldots$ & $\ldots$ \\
SN 2011cl & $\ldots$ & $\ldots$ & $-0.20(0.05)$ \\
SN 2013cf & $\ldots$ & $\ldots$ & $1.49(0.02)$ \\
SN 2013ej & $4.24(0.06)$ & $2.23(0.03)$ & \\
\hline & & & $\ldots$ \\
& & $\ldots$ & $\ldots$ \\
\end{tabular}

continuum in several observed and physical properties, some continuous parameter(s) has(have) to be responsible for such a sequence.

With this aim, we looked at correlations between SFR intensity, oxygen abundance, and $\mathrm{H} \alpha \mathrm{EW}$ measured at the SN II locations and $B V R$ broadband light-curve parametrization defined in Anderson et al. (2014) for those objects in PISCO with available light curves to check if any environmental parameters might be behind that sequence. In Figure 10, we show how the post-maximum brightness decline $(s)$ relates to these local parameters, and all relevant numbers are presented in Table 5.

Only the correlation between $s$ and $\Sigma_{\mathrm{SFR}}$ is significant in all three bands according to a Pearson test (factors of $0.82,0.81$, and 0.73 in the $B, V$, and $R$ bands, respectively). ${ }^{28}$ Lower correlation factors (from 0.1 in $B$ to 0.4 in $R$ ) were found for the $\mathrm{H} \alpha \mathrm{EW}$, and no correlation $(\sim 0.0$ in the $V$ and $R$ bands, and 0.3 in the $B$ band) was found for $\mathrm{O} / \mathrm{H}$, in agreement with the results presented in Anderson et al. (2016). When using the brightness decline parameter during the plateau phase $\left(s_{2}\right)$, while the significance in the $\Sigma_{\mathrm{SFR}}$ relation decreases to $(B, V$, $R)=(0.50,0.75,0.67)$, the relation with $\mathrm{EW}\left(\mathrm{H}_{\alpha}\right)$ increases its significance to $(B, V, R)=(0.56,0.34,0.49){ }^{29}$

The dependence of the post-maximum brightness decline on the local SFR intensity has never been studied before, and may be key in explaining the diversity of the slopes in SNe II: fastdeclining SNe II, traditionally labeled as IIL, would occur at regions with denser SFR compared to slow-declining SNe II, traditionally labeled as IIP. Our results would disfavor different channels for different SNe II, and instead differences may be explained by other continuous properties proxied by $\Sigma_{\mathrm{SFR}}$.

\footnotetext{
28 The relation is not driven by the point at high $\Sigma_{\mathrm{SFR}}$ as shown by the still high correlation factors found when this point is not considered: $0.69,0.49$, and 0.50 , respectively, for the $B V R$ bands.

29 While $s$ accounts for the brightness decline rate from the epoch maximum light to the end of the plateau phase, $s_{2}$ takes into account that in some SNe II the transition between the cooling and the recombination phases becomes evident as a variation (slowdown) of the brightness decline. While $s$ contains information from both phases, $s_{2}$ purely relates to the amount of hydrogen in the outer layer.
} 
Given that the SFR was measured using the local $\mathrm{H} \alpha$ flux as a proxy, and therefore it is tracing very recent and ongoing SF (up to a few tens of Myr), this correlation may also indicate a sequence in progenitor age lifetimes, in the sense that fast decliners would come from younger progenitors than those of slow decliners. This is in line with the result from Anderson et al. (2012), where SN IIL locations were shown to be more correlated to the distribution of SF in their hosts than the locations of $\mathrm{SNe}$ IIP, as shown in their NCR diagram constructed from $\mathrm{H} \alpha$ narrowband imaging.

However, given the small number of objects used here, this trend needs further confirmation with more data. This is the goal of a specific ongoing work combining PISCO with other IFS data, and will be presented elsewhere.

\section{Summary and Conclusions}

In this paper, we presented PISCO, an ongoing effort to complete the SN host galaxy sample with IFU observations selected from the CALIFA survey including low-mass CCSN hosts and objects with SN Ia light curves available. These observations cover the extent of the whole galaxy, including the SN local environment, and they are all observed with the same instrumental configuration. PISCO consists of 466,347 spectra of 232 host galaxies that hosted $272 \mathrm{SNe}$ of all types, with an average spatial resolution of $380 \mathrm{pc}$, and where we were able to detect $11,270 \mathrm{H}$ II regions.

While the SN Ia host galaxy sample in PISCO is statistically consistent in stellar mass with SN Ia hosts from untargeted surveys, CCSNe hosts in PISCO are still slightly biased toward high-mass galaxies, a characteristic of targeted searches. However, this bias has been reduced compared to the CCSN host sample presented in Galbany et al. (2016b). We constructed the distributions of SFR intensity, $\mathrm{EW}(\mathrm{H} \alpha)$, light-weighted average stellar age, and $\mathrm{O} / \mathrm{H}$ measured at $\mathrm{SN}$ locations and recovered previous sequences in terms of the association of these parameters with different SN types. We reconstructed the SFHs at all SN locations by dividing the contributions of young SSPs into four bins between 0 and $300 \mathrm{Myr}$. For the first time, H II region distributions of several parameters were constructed for each galaxy, in order to study how the SN parent region was ranked within the $\mathrm{H}$ II regions of the same galaxy. Then, we averaged out all parameters and ranks for each $\mathrm{SN}$ type. Our main results are as follows.

1. We found that $\mathrm{SNe}$ Ic occur at higher SFR, higher $\mathrm{EW}(\mathrm{H} \alpha)$, and more metal-rich $\mathrm{H}$ II regions within their hosts, as compared to the mean values within those same galaxies. SN Ic environments need a larger fraction of young (0-40 Myr) SSP models to reproduce their spectra than other CCSN types which, together with the highest association of this $\mathrm{SN}$ type to $\Sigma_{\mathrm{SFR}}$ environments and the higher $\mathrm{EW}(\mathrm{H} \alpha)$ found at their locations, supports the picture of SNe Ic having more massive progenitors than other CCSNe.

2. SNe IIb environments are the most different compared to other CCSN types, both in general and within their host galaxies. $\mathrm{SNe}$ IIb occur in metal-poor, low $\mathrm{EW}(\mathrm{H} \alpha)$, and relatively low-SFR environments. They also have the smallest component of young SSP in the innermost bin of the SFH at their locations, and the highest (with SNe IIn) in the oldest bin. They would perfectly fit in the binary system progenitor scenario of two lower-mass stars.
3. $\mathrm{SNe} \mathrm{Ib}$ fall in between $\mathrm{SNe} \mathrm{Ic}$ and $\mathrm{SNe} \mathrm{IIb}$ in all of our diagrams. We suggest that both scenarios, single stars with weaker stellar winds as compared to SNe Ic (given that the $\mathrm{SNe} \mathrm{Ic} / \mathrm{Ib}$ increases with metallicity) and binary stars with stronger mass transfer than for SNe IIb, may be at play.

4. We detect an excess of both very young and old SSPs in the SFHs of SN IIn environments compared to other SN types. This would support the picture of different progenitors for both components: LBVs for the young component, and RSGs for the old component.

5. We present for the first time a correlation between the observed light-curve parameters and local environmental properties of SNe II. In particular, we found that the SN II post-maximum brightness decline in all three $B V R$ bands shows a trend with the local SFR intensity, which could explain the range of decline rates of fast-declining (IIL) and slow-declining (IIP) SNe II, and indicate a difference in the progenitor age lifetimes: lower ages and therefore higher masses for fast-declining SN IIL as compared to slower declining SNe IIP.

6. Our results highlight the need to construct samples of IFS observations built from unbiased survey(s) of $\mathrm{SNe}$, also volume-limited, to get reliable constraints on the associations of different SN types with environmental properties.

PISCO-reduced datacubes and data products generated during the analysis, such as emission-line maps, H II region segregation maps, and $\mathrm{H}$ II region spectra, are made publicly available from the PISCO GitHub repository ${ }^{30}$ and archived in Zenodo (doi:10.5281/zenodo.1172156). With this paper, we release all CCSN host galaxies, while current SN Ia hosts data set will be published in a forthcoming paper. PISCO is an ongoing project focused on different aspects of supernova science, and future publications will be accompanied by the release of the specific data set used with that goal.

\section{Future Work}

One important caveat in the distinction of objects discovered by targeted/untargeted surveys is that, although untargeted surveys discover transients unbiasedly in terms of galaxy properties, given the large amount of objects detected and the limited resources for classification and follow up, there could still be some bias in the object prioritization. Therefore, it is important to construct an SN sample that is both unbiased in terms of discovery but also with a clear constraint on follow-up priority. We anticipate that in the near future, in order to improve current environmental analyses and to alleviate biases such as the host galaxy mass shown in Section 4, it would be beneficial to define homogeneous samples of SNe discovered blindly. The Large Synoptic Survey Telescope (LSST; LSST Science Collaboration et al. 2017) and the Zwicky Transient Factory (ZTF; Bellm et al. 2015) open an opportunity as feeder surveys for an IFU blind follow-up program for both CCSNe and $\mathrm{SNe}$ Ia which, in the case of SNe Ia, would be an excellent low- $z$ companion survey for the Wide-Field InfraRed Survey Telescope (Spergel et al. 2015) SN survey. ${ }^{31}$

\footnotetext{
30 https://github.com/lgalbany/pisco

${ }^{31}$ Assuming that the Integral Field Unit Channel (IFC) is included in the final design of the instrument.
} 
To improve spatial resolution, we have started a new effort, the All-weather MUse Supernova Integral-field Nearby Galaxies (AMUSING; Galbany et al. 2016a) survey that takes advantage of the combination of the wide-aperture $8.1 \mathrm{~m}$ Very Large Telescope at Cerro Paranal, and the Multi-Unit Spectroscopic Explorer (MUSE; Bacon et al. 2014) Integral Field Unit, which provides a large FoV $\left(1^{\prime} \times 1^{\prime}\right)$ and an impressive spatial resolution $(0 . / 2 \times 0$.! 2 per spaxel $)$ only limited by seeing. With almost 100,000 spectra from $~ 4650-9000 \AA$ and $R \sim 3500-1800$ (from the blue to the red extent) delivered per pointing, the field of $\mathrm{SN}$ environments has entered the regime of big data. Currently, after five semesters collecting data, the AMUSING sample consists of more than 300 nearby SN host galaxies, it has already delivered a few analyses of both galaxy enrichment and evolution (Sánchez et al. 2015; Sánchez-Menguiano et al. 2016, 2018; Lopez-Coba et al. 2017) and SN environments (Galbany et al. 2016a; Kuncarayakti et al. 2016, 2017; Prieto et al. 2016; Krühler et al. 2017), and will certainly revolutionize the field in the coming years.

We are greatly in debt to the Calar Alto Observatory staff because without their kindness and interest for this project, most of the observations would have not been possible. We are greatly thankful to Yao-Yuan Mao for being behind most of the Python coding developed for plotting our results, as well as to Or Graur for useful discussions. L.G. and W.M.W.-V. were supported in part by the US National Science Foundation under Grant AST-1311862. I.D. is funded by the MINECO-FEDER AYA2015-63588-P grant. Escrit en la seva major part a cavall de la Biblioteca de Palafrugell i la Platgeta de Calella.

Based on observations collected at the Centro Astronómico Hispano Alemán (CAHA) at Calar Alto, operated jointly by the Max-Planck-Institut für Astronomie (MPIA) and the Instituto de Astrofísica de Andalucía (CSIC). This study makes use of the data provided by the Calar Alto Legacy Integral Field Area (CALIFA) survey (http://www.caha.es/CALIFA/). The STARLIGHT project is supported by the Brazilian agencies CNPq, CAPES, and FAPESP, and by the France-Brazil CAPES/Cofecub program. This research has made use of the NASA/IPAC Extragalactic Database (NED), which is operated by the Jet Propulsion Laboratory, California Institute of Technology, under contract with the National Aeronautics and Space Administration, and data products from the SDSS and Pan-STARRS surveys. Funding for the Sloan Digital Sky Survey IV has been provided by the Alfred P. Sloan Foundation, the U.S. Department of Energy Office of Science, and the Participating Institutions. SDSS-IV acknowledges support and resources from the Center for High-Performance Computing at the University of Utah. The SDSS Web site is http://www.sdss.org. SDSS-IV is managed by the Astrophysical Research Consortium for the Participating Institutions of the SDSS Collaboration including the Brazilian Participation Group, the Carnegie Institution for Science, Carnegie Mellon University, the Chilean Participation Group, the French Participation Group, Harvard-Smithsonian Center for Astrophysics, Instituto de Astrofísica de Canarias, The Johns Hopkins University, Kavli Institute for the Physics and Mathematics of the Universe (IPMU)/University of Tokyo, Lawrence Berkeley National Laboratory, Leibniz Institut für Astrophysik Potsdam (AIP), Max-Planck-Institut für Astronomie (MPIA Heidelberg), Max-Planck-Institut für Astrophysik (MPA Garching), Max-Planck-Institut für Extraterrestrische
Physik (MPE), National Astronomical Observatories of China, New Mexico State University, New York University, University of Notre Dame, Observatário Nacional/MCTI, The Ohio State University, Pennsylvania State University, Shanghai Astronomical Observatory, United Kingdom Participation Group, Universidad Nacional Autónoma de México, University of Arizona, University of Colorado Boulder, University of Oxford, University of Portsmouth, University of Utah, University of Virginia, University of Washington, University of Wisconsin, Vanderbilt University, and Yale University. The Pan-STARRS1 Surveys (PS1) and the PS1 public science archive have been made possible through contributions by the Institute for Astronomy, the University of Hawaii, the PanSTARRS Project Office, the Max-Planck Society and its participating institutes, the Max Planck Institute for Astronomy, Heidelberg, and the Max Planck Institute for Extraterrestrial Physics, Garching, The Johns Hopkins University, Durham University, the University of Edinburgh, the Queen's University Belfast, the Harvard-Smithsonian Center for Astrophysics, the Las Cumbres Observatory Global Telescope Network Incorporated, the National Central University of Taiwan, the Space Telescope Science Institute, the National Aeronautics and Space Administration under grant No. NNX08AR22G issued through the Planetary Science Division of the NASA Science Mission Directorate, the National Science Foundation grant No. AST-1238877, the University of Maryland, Eotvos Lorand University (ELTE), the Los Alamos National Laboratory, and the Gordon and Betty Moore Foundation.

Facility: CAO:3.5m(PMAS/PPak).

Software: Numpy (Van Der Walt et al. 2011), Matplotlib (Hunter 2007), STARLIGHT (Cid Fernandes et al. 2005), HIIexplorer (Sánchez et al. 2012b).

\section{Appendix A Description of Previous Observations}

Around half of the observations in PISCO have been collected from the following sources:

(i) 115 galaxies that hosted $128 \mathrm{SNe}$ were either included in the CALIFA third data release or observed under a few parallel programs led by CALIFA members that were labeled together as CALIFA-extensions. More details about these CALIFA-extensions are described in the CALIFA third data release (Sánchez et al. 2016).

(ii) Two galaxies, NGC 5668 (Marino et al. 2012) and NGC 3982, were observed under two programs that aimed to study these two particular objects (PI: Marino). They hosted three $\mathrm{SNe}$ in total.

(iii) Four galaxies, NGC 0105, UGC 04008, CGCG 207-042, and UGC 05129, were included in a pilot project to study SN Ia host galaxies with IFS (Stanishev et al. 2012).

(iv) Eight objects are observations from the PPak IFS Nearby Galaxies Survey (PINGS; Rosales-Ortega et al. 2010). These include a few large ( $>12$ pointing) mosaics of nearby wide-field galaxies (e.g., NGC 0628)

Subsamples of galaxies (81, 115, and 23, respectively) from the above sources were used in Galbany et al. (2014, 2016c, 2017), where the properties of the SN local environment in terms of star formation, metallicity, and extinction, respectively, were studied. 
Table 6

Summary of the Time Scheduled and Executed for Each of the Observing Programs Listed in Table 1

\begin{tabular}{|c|c|c|c|c|c|}
\hline $\begin{array}{l}\text { Night } \\
\text { (AAMMDD) }\end{array}$ & $\begin{array}{l}\text { Observed } \\
\text { Objects }\end{array}$ & $\begin{array}{l}\text { Frac. } \\
\text { Night }\end{array}$ & Success & Scheduled & Completion \\
\hline \multicolumn{6}{|c|}{ H15-3.5-004 } \\
\hline 150810 & 4 & 1.00 & 1.00 & 1.00 & $8 \%$ \\
\hline 150811 & 0 & 1.00 & 0.00 & 1.00 & $8 \%$ \\
\hline 151102 & 0 & 1.00 & 0.00 & 1.00 & $8 \%$ \\
\hline 151103 & 1 & 1.00 & 0.09 & 1.09 & $10 \%$ \\
\hline 151218 & 10 & 1.00 & 1.00 & 1.09 & $30 \%$ \\
\hline 151219 & 10 & 1.00 & 1.00 & 2.09 & $50 \%$ \\
\hline 151220 & 10 & 1.00 & 0.91 & 3.00 & $70 \%$ \\
\hline 151221 & 10 & 1.00 & 1.00 & 4.00 & $\mathbf{9 0} \%$ \\
\hline \multicolumn{6}{|c|}{ F16-3.5-006 } \\
\hline 160118 & 1 & 1.00 & 0.23 & 0.23 & $3 \%$ \\
\hline 160119 & 0 & 1.00 & 0.00 & 0.23 & $3 \%$ \\
\hline 160201 & 3 & 0.45 & 0.45 & 0.68 & $13 \%$ \\
\hline 160314 & 4 & 0.75 & 0.67 & 1.33 & $27 \%$ \\
\hline 160316 & 4 & 0.91 & 0.60 & 1.94 & $40 \%$ \\
\hline 160606 & 1 & 0.50 & 0.50 & 2.44 & $43 \%$ \\
\hline 160607 & 5 & 1.00 & 1.00 & 3.44 & $60 \%$ \\
\hline 160608 & 3 & 0.75 & 0.75 & 4.19 & $\mathbf{7 0} \%$ \\
\hline \multicolumn{6}{|c|}{ H16-3.5-012 } \\
\hline 161008 & 2 & 0.17 & 0.05 & 0.05 & $13 \%$ \\
\hline 161009 & 7 & 1.00 & 1.00 & 1.05 & $\mathbf{5 6} \%$ \\
\hline \multicolumn{6}{|c|}{ F17-3.5-001 } \\
\hline 170428 & 0 & 1.00 & 0.00 & 0.00 & $0 \%$ \\
\hline 170429 & 0 & 1.00 & 0.00 & 0.00 & $0 \%$ \\
\hline 170430 & 5 & 1.00 & 1.00 & 1.00 & $25 \%$ \\
\hline 170525 & 3 & 0.45 & 0.45 & 1.45 & $40 \%$ \\
\hline 170526 & 4 & 0.58 & 0.58 & 2.03 & $60 \%$ \\
\hline \multicolumn{6}{|c|}{ H17-3.5-001 } \\
\hline 170817 & 6 & 1.00 & 1.00 & 1.00 & $50 \%$ \\
\hline 170818 & 7 & 1.00 & 1.00 & 2.00 & $100 \%$ \\
\hline
\end{tabular}

Note. The last column tracks the completion percent of the initial program. The numbers in bold represent the percentage of observations completed at the end of each semester with respect to the proposed program.

\section{Appendix B Description of New Observations}

In Table 6, we list the details of all nights dedicated to each of the five programs in PISCO indicating the success ratio. Note that due to unfortunate bad weather nights, the observatory was usually able to award additional nights at the end of the semester that contributed to finishing or increasing the success ratio of the observations significantly. Below, we give more details about each program.

15B: We proposed to observe a sample of galaxies that hosted CCSNe with precise spectral type and that occurred in low-mass galaxies. This allowed us both to partially correct the bias in the host galaxy mass present in the CALIFA SN host galaxy sample and to split the CCSN group into different SN subtypes. This paper presented the results of this particular program.

16A: $\mathrm{SNe}$ Ia are used as accurate distance indicators; however, the nature of their progenitors is still unclear.
Differentiating between the single-degenerate (WD and nondegenerate donor star) and double-degenerate (two WDs) scenarios is a key issue. The detection of blueshifted $\mathrm{NaD}$ absorption in some SN Ia spectra, and its potential association with circumstellar material structures close to the SN progenitor, has been the focus of recent attention. In semester 16A, we proposed the observation of 30 galaxies (of which 21 were actually observed) that hosted $\mathrm{SNe}$ with deep $\mathrm{NaD}$ features detected in their spectra to constrain the nature of the $\mathrm{NaD}$ absorption, and $\mathrm{SN}$ progenitor age and metallicity. We aimed to understand the origin and nature of narrow $\mathrm{NaD}$ absorption in mid-resolution SN Ia spectra, and its relation to either ISM or CSM through PMAS observations of their host galaxies. The sample were selected from works reporting the presence of strong $\mathrm{NaD}$ features in $\mathrm{SN}$ spectra (basically Sternberg et al. 2011; Maguire et al. 2013, and Phillips et al. 2013).

16B/17A/17B: Although optical observations of SNe Ia have proved essential for measuring accurate cosmological distances, they are superior standard candles in the near-infrared (NIR), both because their light curves are intrinsically more similar at these wavelengths, and reddening effects are greatly reduced (Krisciunas et al. 2004; Wood-Vasey et al. 2008). SN Ia Hubble residuals using optical data correlate with global host galaxy parameters (such as total mass), and the addition of a term in the SN Ia light-curve standardization accounting for these environmental parameters has proved to reduce further the scatter in the SN Ia absolute magnitude at peak. We proposed observations of galaxies that hosted SNe Ia that were included in the SweetSpot Survey, a three-year NOAO Survey program that obtained NIR $(J H K)$ observations of 114 nearby $(0.02<z<0.09)$ SNe Ia with the WIYN High-resolution Infrared Camera (WHIRC) on the WIYN $3.5 \mathrm{~m}$ telescope (Weyant et al. 2014, 2017). Thirty-four of the 49 proposed observations $(9 / 16,12 / 20$, and $13 / 13$ per semester respectively) were successfully performed. Our aim using PISCO data is to complement IFS observations from HexPak (Ponder 2017) and MUSE (Galbany et al. 2016a), and reduce further the scatter in the NIR SN Ia Hubble diagram by looking for the first time for correlations between SN Ia residuals and both global and local galactic properties.

\section{ORCID iDs}

L. Galbany (i) https://orcid.org/0000-0002-1296-6887

H. Kuncarayakti (1) https://orcid.org/0000-0002-1132-1366

V. Stanishev (iD https://orcid.org/0000-0002-7626-1181

M. E. Moreno-Raya (iD https://orcid.org/0000-0003-

3437-1339

W. M. Wood-Vasey (D) https://orcid.org/0000-00017113-1233

K. A. Ponder (iD https://orcid.org/0000-0002-8207-3304

C. Badenes (iD https://orcid.org/0000-0003-3494-343X

F. F. Rosales-Ortega (iD https://orcid.org/0000-00023642-9146

R. A. Marino (DD https://orcid.org/0000-0002-8559-6565

\section{References}

Abolfathi, B., Aguado, D. S., Aguilar, G., et al. 2017, arXiv:1707.09322 Anderson, J. P., González-Gaitán, S., Hamuy, M., et al. 2014, ApJ, 786, 67 Anderson, J. P., Gutiérrez, C. P., Dessart, L., et al. 2016, A\&A, 589, A110 Anderson, J. P., Habergham, S. M., James, P. A., \& Hamuy, M. 2012, MNRAS, 424, 1372 
Anderson, J. P., James, P. A., Förster, F., et al. 2015a, MNRAS, 448, 732

Anderson, J. P., James, P. A., Habergham, S. M., Galbany, L., \& Kuncarayakti, H. 2015b, PASA, 32, e019

Arcavi, I., Gal-Yam, A., Kasliwal, M. M., et al. 2010, ApJ, 721, 777

Arnett, W. D., Bahcall, J. N., Kirshner, R. P., \& Woosley, S. E. 1989, ARA\&A, 27, 629

Bacon, R., Vernet, J., Borisova, E., et al. 2014, Msngr, 157, 13

Baldwin, J. A., Phillips, M. M., \& Terlevich, R. 1981, PASP, 93, 5

Barbon, R., Cappellaro, E., \& Turatto, M. 1989, A\&AS, 81, 421

Barbon, R., Ciatti, F., \& Rosino, L. 1979, A\&A, 72, 287

Becker, S. A., \& Iben, I., Jr. 1980, ApJ, 237, 111

Bellm, E. C., Kulkarni, S. R. \& ZTF Collaboration 2015, AAS Meeting, 225, 328.04

Bersten, M. C., Benvenuto, O. G., Folatelli, G., et al. 2014, AJ, 148, 68

Bethe, H. A., Brown, G. E., Applegate, J., \& Lattimer, J. M. 1979, NuPhA, 324,487

Betoule, M., Kessler, R., Guy, J., et al. 2014, A\&A, 568, A22

Bianchi, S., \& Schneider, R. 2007, MNRAS, 378, 973

Bruzual, G. 2007, in ASP Conf. Ser. 374, From Stars to Galaxies: Building the Pieces to Build Up the Universe, ed. A. Vallenari et al. (San Francisco, CA: ASP), 303

Bruzual, G., \& Charlot, S. 2003, MNRAS, 344, 1000

Cappellari, M., \& Copin, Y. 2003, MNRAS, 342, 345

Cardelli, J. A., Clayton, G. C., \& Mathis, J. S. 1989, ApJ, 345, 245

Catalán-Torrecilla, C., Gil de Paz, A., Castillo-Morales, A., et al. 2015, A\&A, 584, A87

Chabrier, G. 2003, PASP, 115, 763

Chambers, K. C., Magnier, E. A., Metcalfe, N., et al. 2016, arXiv:1612.05560

Charbonnel, C., Meynet, G., Maeder, A., Schaller, G., \& Schaerer, D. 1993, A\&AS, 101, 415

Cid Fernandes, R., Mateus, A., Sodré, L., Stasińska, G., \& Gomes, J. M. 2005, MNRAS, 358, 363

Cid Fernandes, R., Schoenell, W., Gomes, J. M., et al. 2009, RMxAC, 35, 127

Cid Fernandes, R., Stasińska, G., Mateus, A., \& Vale Asari, N. 2011, MNRAS, 413, 1687

Claeys, J. S. W., de Mink, S. E., Pols, O. R., Eldridge, J. J., \& Baes, M. 2011, A\&A, 528, A131

Crowther, P. A. 2007, ARA\&A, 45, 177

de Jaeger, T., Galbany, L., Filippenko, A. V., et al. 2017, MNRAS, 472, 4233

Diaz, A. I., Terlevich, E., Pagel, B. E. J., Vilchez, J. M., \& Edmunds, M. G. 1987, MNRAS, 226, 19

Dominguez, I., Chieffi, A., Limongi, M., \& Straniero, O. 1999, ApJ, 524, 226

Dopita, M. A., Kewley, L. J., Sutherland, R. S., \& Nicholls, D. C. 2016, Ap\&SS, 361, 61

Eldridge, J. J., \& Maund, J. R. 2016, MNRAS, 461, L117

Eldridge, J. J., \& Stanway, E. R. 2009, MNRAS, 400, 1019

Elias-Rosa, N., Pastorello, A., Benetti, S., et al. 2016, MNRAS, 463, 3894

Falcón-Barroso, J., Sánchez-Blázquez, P., Vazdekis, A., et al. 2011, A\&A, 532, A95

Faran, T., Poznanski, D., Filippenko, A. V., et al. 2014, MNRAS, 445, 554

Fitzpatrick, E. L. 1999, PASP, 111, 63

Folatelli, G., Bersten, M. C., Benvenuto, O. G., et al. 2014, ApJL, 793, L22

Folatelli, G., Bersten, M. C., Kuncarayakti, H., et al. 2015, ApJ, 811, 147

Folatelli, G., Van Dyk, S. D., Kuncarayakti, H., et al. 2016, ApJL, 825, L22

Fransson, C., Chevalier, R. A., Filippenko, A. V., et al. 2002, ApJ, 572, 350

Galbany, L. 2018, PISCO: The PMAS/PPak Integral field Supernova hosts COmpilation, Zenodo, doi:10.5281/zenodo.1172156

Galbany, L., Anderson, J. P., Rosales-Ortega, F. F., et al. 2016a, MNRAS, 455,4087

Galbany, L., Hamuy, M., Phillips, M. M., et al. 2016b, AJ, 151, 33

Galbany, L., Mora, L., González-Gaitán, S., et al. 2017, MNRAS, 468, 628

Galbany, L., Stanishev, V., Mourão, A. M., et al. 2014, A\&A, 572, A38

Galbany, L., Stanishev, V., Mourão, A. M., et al. 2016c, A\&A, 591, A48

Gal-Yam, A., \& Leonard, D. C. 2009, Natur, 458, 865

Gal-Yam, A., Mazzali, P., Ofek, E. O., et al. 2009, Natur, 462, 624

García-Benito, R., Pérez, E., Díaz, Á. I., Maíz Apellániz, J., \& Cerviño, M. 2011, AJ, 141, 126

García-Benito, R., Zibetti, S., Sánchez, S. F., et al. 2015, A\&A, 576, A135

Georgy, C., Meynet, G., Walder, R., Folini, D., \& Maeder, A. 2009, A\&A, 502,611

Girardi, L., Bressan, A., Bertelli, G., \& Chiosi, C. 2000, A\&AS, 141, 371

González Delgado, R. M., Cerviño, M., Martins, L. P., Leitherer, C., \& Hauschildt, P. H. 2005, MNRAS, 357, 945

González Delgado, R. M., García-Benito, R., Pérez, E., et al. 2015, A\&A, 581, A103

Gonzalez Delgado, R. M., \& Perez, E. 1997, ApJS, 108, 199
González-Gaitán, S., Tominaga, N., Molina, J., et al. 2015, MNRAS, 451,2212

Graur, O., Bianco, F. B., Huang, S., et al. 2017, ApJ, 837, 120

Guillochon, J., Parrent, J., Kelley, L. Z., \& Margutti, R. 2017, ApJ, 835, 64

Habergham, S. M., Anderson, J. P., James, P. A., \& Lyman, J. D. 2014, MNRAS, 441, 2230

Hamuy, M., Phillips, M. M., Suntzeff, N. B., et al. 2003, Natur, 424, 651

Heger, A., Fryer, C. L., Woosley, S. E., Langer, N., \& Hartmann, D. H. 2003 , ApJ, 591, 288

Horne, K. 1986, PASP, 98, 609

Hoyle, F., \& Fowler, W. A. 1960, ApJ, 132, 565

Hunter, J. D. 2007, CSE, 9, 90

Husemann, B., Jahnke, K., Sánchez, S. F., et al. 2013, A\&A, 549, A87

Jerkstrand, A. 2017, arXiv:1702.06702

Kangas, T., Portinari, L., Mattila, S., et al. 2017, A\&A, 597, A92

Kasen, D., \& Bildsten, L. 2010, ApJ, 717, 245

Kelly, P. L., Filippenko, A. V., Burke, D. L., et al. 2015, Sci, 347, 1459

Kelly, P. L., \& Kirshner, R. P. 2012, ApJ, 759, 107

Kelz, A., Verheijen, M. A. W., Roth, M. M., et al. 2006, PASP, 118, 129

Kennicutt, R. C., Jr. 1998a, ARA\&A, 36, 189

Kennicutt, R. C., Jr. 1998b, ApJ, 498, 541

Kewley, L. J., Dopita, M. A., Sutherland, R. S., Heisler, C. A., \& Trevena, J. 2001, ApJ, 556, 121

Krisciunas, K., Suntzeff, N. B., Phillips, M. M., et al. 2004, AJ, 128, 3034

Krühler, T., Kuncarayakti, H., Schady, P., et al. 2017, A\&A, 602, A85

Kuncarayakti, H., Anderson, J. P., Galbany, L., et al. 2017, A\&A, in press (arXiv:1711.05765)

Kuncarayakti, H., Doi, M., Aldering, G., et al. 2013a, AJ, 146, 30

Kuncarayakti, H., Doi, M., Aldering, G., et al. 2013b, AJ, 146, 31

Kuncarayakti, H., Galbany, L., Anderson, J. P., Krühler, T., \& Hamuy, M. 2016, A\&A, 593, A78

Kuncarayakti, H., Maeda, K., Ashall, C. J., et al. 2018, ApJL, 854, L14

Kuncarayakti, H., Maeda, K., Bersten, M. C., et al. 2015a, A\&A, 579, A95

Kuncarayakti, H., Maeda, K., Bersten, M. C., et al. 2015b, A\&A, 579, A95

Leitherer, C., Schaerer, D., Goldader, J. D., et al. 1999, ApJS, 123, 3

Lopez, L. A., Krumholz, M. R., Bolatto, A. D., Prochaska, J. X., \& Ramirez-Ruiz, E. 2011, ApJ, 731, 91

Lopez-Coba, C., Sanchez, S. F., Cruz-Gonzalez, I., et al. 2017, ApJL, 850, L17

López-Sánchez, Á R., Dopita, M. A., Kewley, L. J., et al. 2012, MNRAS, 426, 2630

López-Sánchez, A. R., \& Esteban, C. 2009, A\&A, 508, 615

López-Sánchez, Á R., \& Esteban, C. 2010, A\&A, 516, A104

LSST Science Collaboration, Marshall, P., Anguita, T., et al. 2017, arXiv:1708. 04058

Lyman, J. D., Taddia, F., Stritzinger, M. D., et al. 2018, MNRAS, 473, 1359

Mackey, J., Mohamed, S., Gvaramadze, V. V., et al. 2014, Natur, 512, 282

Maguire, K., Sullivan, M., Patat, F., et al. 2013, MNRAS, 436, 222

Maoz, D., Mannucci, F., \& Nelemans, G. 2014, ARA\&A, 52, 107

Marigo, P., \& Girardi, L. 2007, A\&A, 469, 239

Marigo, P., Girardi, L., Bressan, A., et al. 2008, A\&A, 482, 883

Marino, R. A., Gil de Paz, A., Castillo-Morales, A., et al. 2012, ApJ, 754, 61

Marino, R. A., Rosales-Ortega, F. F., Sánchez, S. F., et al. 2013, A\&A, 559, A114

Martig, M., \& Bournaud, F. 2010, ApJL, 714, L275

Mast, D., Rosales-Ortega, F. F., Sánchez, S. F., et al. 2014, A\&A, 561, A129

Matteucci, F., \& Greggio, L. 1986, A\&A, 154, 279

Mauerhan, J. C., Smith, N., Filippenko, A. V., et al. 2013, MNRAS, 430, 1801

Maund, J. R., Smartt, S. J., Kudritzki, R. P., Podsiadlowski, P., \& Gilmore, G. F. 2004, Natur, 427, 129

Moustakas, J., Kennicutt, R. C., Jr., Tremonti, C. A., et al. 2010, ApJS, 190, 233

Neill, J. D., Sullivan, M., Howell, D. A., et al. 2009, ApJ, 707, 1449

Osterbrock, D. E., \& Ferland, G. J. 2006, Astrophysics of Gaseous Nebulae and Active Galactic Nuclei (Mill Valley, CA: Univ. Science Books)

Pan, Y.-C., Sullivan, M., Maguire, K., et al. 2014, MNRAS, 438, 1391 Pastorello, A., Cappellaro, E., Inserra, C., et al. 2013, ApJ, 767, 1

Pastorello, A., Mattila, S., Zampieri, L., et al. 2008, MNRAS, 389, 113

Peimbert, M., \& Costero, R. 1969, BOTT, 5, 3

Peimbert, M., Peimbert, A., Esteban, C., et al. 2007, RMxAC, 29, 72

Pettini, M., \& Pagel, B. E. J. 2004, MNRAS, 348, L59

Phillips, M. M., Simon, J. D., Morrell, N., et al. 2013, ApJ, 779, 38

Podsiadlowski, P., Joss, P. C., \& Hsu, J. J. L. 1992, ApJ, 391, 246

Ponder, K. A. 2017, PhD dissertation, Univ. Pittsburgh

Prieto, J. L., Krühler, T., Anderson, J. P., et al. 2016, ApJL, 830, L32

Rigault, M., Copin, Y., Aldering, G., et al. 2013, A\&A, 560, 66

Roman, M., Hardin, D., Betoule, M., et al. 2017, arXiv:1706.07697 
Rosales-Ortega, F. F., Kennicutt, R. C., Sánchez, S. F., et al. 2010, MNRAS, 405, 735

Roth, M. M., Kelz, A., Fechner, T., et al. 2005, PASP, 117, 620

Ryder, S., Staveley-Smith, L., Dopita, M., et al. 1993, ApJ, 416, 167

Salpeter, E. E. 1955, ApJ, 121, 161

Sana, H., de Mink, S. E., de Koter, A., et al. 2012, Sci, 337, 444

Sánchez, S. F., Galbany, L., Pérez, E., et al. 2015, A\&A, 573, A105

Sánchez, S. F., García-Benito, R., Zibetti, S., et al. 2016, A\&A, 594, A36

Sánchez, S. F., Kennicutt, R. C., Gil de Paz, A., et al. 2012a, A\&A, 538, A8

Sánchez, S. F., Rosales-Ortega, F. F., Jungwiert, B., et al. 2013, A\&A, 554, A58

Sánchez, S. F., Rosales-Ortega, F. F., Marino, R. A., et al. 2012b, A\&A, 546, A2

Sánchez-Blázquez, P., Peletier, R. F., Jiménez-Vicente, J., et al. 2006, MNRAS, 371, 703

Sánchez-Menguiano, L., Sánchez, S. F., Kawata, D., et al. 2016, ApJL, 830, L40

Sánchez-Menguiano, L., Sánchez, S. F., Pérez, I., et al. 2018, A\&A, 609, A119

Sanders, N. E., Soderberg, A. M., Gezari, S., et al. 2015, ApJ, 799, 208

Schaerer, D., Charbonnel, C., Meynet, G., Maeder, A., \& Schaller, G. 1993a, A\&AS, 102, 339

Schaerer, D., Meynet, G., Maeder, A., \& Schaller, G. 1993b, A\&AS, 98, 523

Schaller, G., Schaerer, D., Meynet, G., \& Maeder, A. 1992, A\&AS, 96, 269

Schlegel, D. J., Finkbeiner, D. P., \& Davis, M. 1998, ApJ, 500, 525

Scolnic, D. M., Jones, D. O., Rest, A., et al. 2017, arXiv:1710.00845

Sellwood, J. A., \& Binney, J. J. 2002, MNRAS, 336, 785

Smartt, S. J. 2015, PASA, 32, e016

Smith, N., Hinkle, K. H., \& Ryde, N. 2009, AJ, 137, 3558

Smith, N., Li, W., Filippenko, A. V., \& Chornock, R. 2011, MNRAS, 412,1522
Smith, N., \& Tombleson, R. 2015, MNRAS, 447, 598

Spergel, D., Gehrels, N., Baltay, C., et al. 2015, arXiv:1503.03757

Stanishev, V., Rodrigues, M., Mourão, A., \& Flores, H. 2012, A\&A, 545, A58

Stasińska, G. 1978, A\&A, 66, 257

Sternberg, A., Gal-Yam, A., Simon, J. D., et al. 2011, Sci, 333, 856

Stoll, R., Prieto, J. L., Stanek, K. Z., \& Pogge, R. W. 2013, ApJ, 773, 12

Swartz, D. A., Wheeler, J. C., \& Harkness, R. P. 1991, ApJ, 374, 266

Taddia, F., Sollerman, J., Fremling, C., et al. 2015, A\&A, 580, A131

Taddia, F., Stritzinger, M. D., Sollerman, J., et al. 2013, A\&A, 555, A10

Tartaglia, L., Fraser, M., Sand, D. J., et al. 2017, ApJL, 836, L12

Todini, P., \& Ferrara, A. 2001, MNRAS, 325, 726

Valenti, S., Howell, D. A., Stritzinger, M. D., et al. 2016, MNRAS, 459, 3939

Valenti, S., Sand, D., Stritzinger, M., et al. 2015, MNRAS, 448, 2608

Van Der Walt, S., Colbert, S. C., \& Varoquaux, G. 2011, arXiv:1102.1523

Van Dyk, S. D., Zheng, W., Fox, O. D., et al. 2014, AJ, 147, 37

Vazdekis, A., Sánchez-Blázquez, P., Falcón-Barroso, J., et al. 2010, MNRAS, 404, 1639

Verheijen, M. A. W., Bershady, M. A., Andersen, D. R., et al. 2004, AN, 325,151

Vilchez, J. M., \& Esteban, C. 1996, MNRAS, 280, 720

Vink, J. S., \& de Koter, A. 2005, A\&A, 442, 587

Vink, J. S., de Koter, A., \& Lamers, H. J. G. L. M. 2001, A\&A, 369, 574

Walcher, C. J., Wisotzki, L., Bekeraité, S., et al. 2014, A\&A, 569, A1

Weyant, A., Wood-Vasey, W. M., Allen, L., et al. 2014, ApJ, 784, 105

Weyant, A., Wood-Vasey, W. M., Joyce, R., et al. 2017, arXiv:1703.02402

Wood-Vasey, W. M., Friedman, A. S., Bloom, J. S., et al. 2008, ApJ, 689, 377

Woosley, S. E., \& Weaver, T. A. 1986, ARA\&A, 24, 205

Yoon, S.-C. 2015, PASA, 32, e015

Yoon, S.-C. 2017, MNRAS, 470, 3970

Zapartas, E., de Mink, S. E., Izzard, R. G., et al. 2017, A\&A, 601, A29 